\title{
BERNSTEIN POLYNOMIAL OF 2-PUISEUX PAIRS IRREDUCIBLE PLANE CURVE SINGULARITIES
}

\author{
E. ARTAL BARTOLO ${ }^{1}$, PI. CASSOU-NOGUÈS ${ }^{2}$, I. LUENGO ${ }^{3}$, \\ AND A. MELLE-HERNÁNDEZ ${ }^{3}$
}

Dedicated to Prof. H. B. Laufer on the occasion of his 70th birthday

\begin{abstract}
In 1982, Tamaki Yano proposed a conjecture predicting the set of $b$-exponents of an irreducible plane curve singularity germ which is generic in its equisingularity class. In [1] we proved the conjecture for the case in which the germ has two Puiseux pairs and its algebraic monodromy has distinct eigenvalues. In this article we aim to study the Bernstein polynomial for any function with the hypotheses above. In particular the set of all common roots of those Bernstein polynomials is given. We provide also bounds for some analytic invariants of singularities and illustrate the computations in suitable examples.
\end{abstract}

\section{INTRODUCTION}

One of the main guide lines of Prof. H.B. Laufer in singularity theory, particularly concerning normal two dimensional analytic singularities $(X, 0)$, has been which analytic invariants of $(X, 0)$ depends on the topology, i.e., they are characterized by their link $L_{(X, 0)}$. The link has the same information as the decorated resolution graph $\Gamma_{(X ; 0)}$ see [20]. For instance Laufer questioned the following in [13]: What conditions does the existence of a hypersurface representative of $(X, 0)$ put on a decorated dual graph $\Gamma_{(X, 0)}$ ? The analytic properties of $X$ depend on the analytic properties of the ramification locus of a projection. In this work, we study the behavior of some analytic (non-topological) invariants for germs of curves. The main goal of the paper is to consider germs of irreducible plane curve singularities with the same topology and describe exactly the set of common roots of their corresponding local Bernstein polynomials which are analytic invariants of their germs.

2010 Mathematics Subject Classification. Primary 14F10,32S40; Secondary 32S05,32A30.

Key words and phrases. Bernstein polynomial, $b$-exponents, improper integrals.

${ }^{1}$ Partially supported by the grant MTM2013-45710-C02-01-P and Grupo Geometría of Gobierno de Aragón/Fondo Social Europeo.

${ }^{2}$ Partially supported by MTM2013-45710-C02-01-P and MTM2013-45710-C02-02-P.

${ }^{3}$ Partially supported by the grant MTM2013-45710-C02-02-P. 
Let $\mathcal{O}$ be the ring of germs of holomorphic functions on $\left(\mathbb{C}^{n}, 0\right), \mathcal{D}$ the ring of germs of holomorphic differential operators of finite order with coefficients in $\mathcal{O}$. Let $s$ be an indeterminate commuting with the elements of $\mathcal{D}$ and set $\mathcal{D}[s]=$ $\mathcal{D} \otimes_{\mathbb{C}} \mathbb{C}[s]$.

Given an holomorphic germ $f \in \mathcal{O}$, one considers the ring $R_{f, s}:=\mathcal{O}\left[\frac{1}{f}, s\right]$ and the free $R_{f, s}$-module $R_{f, s} f^{s}$ of rank 1 with the natural $\mathcal{D}[s]$-module structure. Then, there exists a non-zero polynomial $B(s) \in \mathbb{C}[s]$ and some differential operator $P=P(s, x, D) \in \mathcal{D}[s]$, holomorphic in $x_{1}, \ldots, x_{n}$ and polynomial in $\frac{\partial}{\partial x_{1}}, \ldots, \frac{\partial}{\partial x_{n}}$, which satisfies in $R_{f, s} f^{s}$ the following functional equation

$$
P(s, x, D) \cdot f(x)^{s+1}=B(s) \cdot f(x)^{s}
$$

The monic generator $b_{f, 0}(s)$ of the ideal of such polynomials $B(s)$ is called the Bernstein polynomial (or $b$-function or Berstein-Sato polynomial) of $f$ at 0 . The same result holds if we replace $\mathcal{O}$ by the ring of polynomials in a characteristic zero field $\mathbb{K}$ with the obvious corrections, see e.g. [8, 10, Theorem 3.3].

This result was first obtained for $f$ polynomial by Bernstein in [2] and in general by Björk [3]. One can prove that $b_{f, 0}(s)$ is divisible by $s+1$, and we consider the reduced Bernstein polynomial $\tilde{b}_{f, 0}(s):=\frac{b_{f, 0}(s)}{s+1}$.

In the case where $f$ defines an isolated singularity, one can consider the Brieskorn lattice $H_{0}^{\prime \prime}:=\Omega^{n} / d f \wedge d \Omega^{n-2}$ and its saturated $\tilde{H}_{0}^{\prime \prime}=\sum_{k \geq 0}\left(\partial_{t} t\right)^{k} H_{0}^{\prime \prime}$. Malgrange [18] showed that the reduced Bernstein polynomial $\tilde{b}_{f, 0}(s)$ is the minimal polynomial of the endomorphism $-\partial_{t} t$ on the vector space $F:=\tilde{H}_{0}^{\prime \prime} / \partial_{t}^{-1} \tilde{H}_{0}^{\prime \prime}$, whose dimension equals the Minor number $\mu(f, 0)$ of $f$ at 0 . The $b$-exponents $\left\{\beta_{1}, \ldots, \beta_{\mu}\right\}$ are the roots of the characteristic polynomial of the endomorphism $\partial_{t} t$. Recall that $\exp \left(-2 i \pi \partial_{t} t\right)$ can be identified with the algebraic monodromy of the Milnor fiber of $f$ at the singular point.

Kashiwara [12] expressed these ideas with differential operators. Let $\mathcal{M}:=$ $\mathcal{D}[s] f^{s} / \mathcal{D}[s] f^{s+1}$, where $s$ defines an endomorphism of $P(s) f^{s}$ by multiplication. This morphism keeps invariant $\tilde{\mathcal{M}}:=(s+1) \mathcal{M}$ and defines a linear endomorphism of $\left(\Omega^{n} \otimes_{\mathcal{D}} \tilde{\mathcal{M}}\right)_{0}$ which is naturally identified with $F$ and under this identification $-\partial_{t} t$ becomes the endomorphism defined by the multiplication by $s$.

In [18, Malgrange proved that the set $R_{f, 0}$ of roots of the Bernstein polynomial is contained in $\mathbb{Q}_{<0}$. Moreover, Kashiwara [12] restricted the set of candidate roots. The number $-\alpha_{f, 0}:=\max R_{f, 0}$ is the opposite of the log canonical threshold of the singularity. Saito [21] proved that

$$
R_{f, 0} \subset\left[-n+\alpha_{f, 0},-\alpha_{f, 0}\right] .
$$


Now let $f$ be an irreducible germ of plane curve. In 1982, Tamaki Yano [29] made a conjecture concerning the $b$-exponents. We state this conjecture in the case we are interested in, the case of two Puiseux pairs. Let $C S_{n_{1}, m}^{n_{2}, q}:=\left(n_{1} n_{2}, m n_{2}, m n_{2}+q\right)$ be the characteristic sequence of $f$, such that

- $1<n_{1}<m, \operatorname{gcd}\left(m, n_{1}\right)=1$;

- $q>0, n_{2}>1, \operatorname{gcd}\left(q, n_{2}\right)=1$.

Recall that this means that $f(x, y)=0$ has as root (say over $x$ ) a Puiseux expansion

$$
x=\cdots+a_{1} y^{\frac{m}{n_{1}}}+\cdots+a_{2} y^{\frac{m n_{2}+q}{n_{1} n_{2}}}+\ldots
$$

with exactly 2 characteristic monomials.

Let

$$
\begin{aligned}
& B_{1}:=\left\{\alpha=\frac{m+n_{1}+k}{m n_{1} n_{2}}: 0 \leq k<m n_{1} n_{2}, \text { and } n_{2} m \alpha, n_{2} n_{1} \alpha \notin \mathbb{Z}\right\} ; \\
& B_{2}:=\{\alpha=\overbrace{\left.\frac{\left(m+n_{1}\right) n_{2}+q+k}{n_{2} \underbrace{\left(m n_{1} n_{2}+q\right)}_{D}} \mid 0 \leq k<n_{2} D \text { and } n_{2} \alpha, D \alpha \notin \mathbb{Z}\right\} .}^{N_{k}} \mid
\end{aligned}
$$

Yano's Conjecture ([29]). For almost all irreducible plane curve singularity germ $f:\left(\mathbb{C}^{2}, 0\right) \rightarrow(\mathbb{C}, 0)$ with characteristic sequence $\left(n_{1} n_{2}, m n_{2}, m n_{2}+q\right)$, the set $B_{f}$ of the b-exponents $\left\{\beta_{1}, \ldots, \beta_{\mu}\right\}$ is $B_{1} \cup B_{2}$.

In [1] Yano's conjecture was proved for the case

$$
\operatorname{gcd}\left(q, n_{1}\right)=1 \text { or } \operatorname{gcd}(q, m)=1 .
$$

The above condition is equivalent to require that the algebraic monodromy of the irreducible germ has distinct eigenvalues. In this case, the $\mu b$-exponents are all distinct and they coincide with the opposite of the roots of the reduced Bernstein polynomial (which turns out to be of degree $\mu$ ).

There is another set which is important too, the set of the exponents of the monodromy (or spectral numbers, up to the shift by one, in the terminology of Varchenko [28]). This notion was first introduced by Steenbrink [25].

Let $f:\left(\mathbb{C}^{n}, 0\right) \longrightarrow(\mathbb{C}, 0)$ be a germ of a holomorphic function with isolated singularity. In [25] Steenbrink constructed a mixed Hodge structure on $H^{n-1}\left(F_{f, 0}, \mathbb{C}\right)$. Let

$$
H^{n-1}\left(F_{f, 0}, \mathbb{C}\right)_{\lambda}=\operatorname{Ker}\left(T_{s}-\lambda: H^{n-1}\left(F_{f, 0}, \mathbb{C}\right) \longrightarrow H^{n-1}\left(F_{f, 0}, \mathbb{C}\right)\right) ;
$$

where $T_{u}, T_{s}$ are, respectively, the unipotent and semisimple factors of the Jordan decomposition of the monodromy $h^{n-1}$. 
The set $\operatorname{Spec}(f)$ of spectral numbers are $\mu$ rational numbers

$$
0<\alpha_{1} \leq \alpha_{2} \leq \cdots \leq \alpha_{\mu}<n
$$

which are defined by the following condition:

$$
\begin{gathered}
\#\left\{j: \exp \left(-2 \pi i \alpha_{j}\right)=\lambda,\left\lfloor\alpha_{j}\right\rfloor=n-p-1\right\}=\operatorname{dim}_{\mathbb{C}} \operatorname{Gr}_{F}^{p} H^{n-1}\left(F_{f, 0}, \mathbb{C}\right)_{\lambda}, \quad \lambda \neq 1 \\
\#\left\{j: \alpha_{j}=n-p\right\}=\operatorname{dim}_{\mathbb{C}} \operatorname{Gr}_{F}^{p} H^{n-1}\left(F_{f, 0}, \mathbb{C}\right)_{1} .
\end{gathered}
$$

The set $\operatorname{Spec}(f)$ of spectral numbers is symmetric, that is $\alpha_{i}+\alpha_{\mu-(i-1)}=n$. It is known that this set is constant under $\mu$-constant deformation of $f$, see [28].

M. Saito 23. gave a formula for $\operatorname{Spec}(f)$ in the case of a germ of an irreducible plane curve singularity (cf. also Theorem 3.1 in [19] or section 2.2 in [24]). In the case of characteristic sequence $\left(n_{1} n_{2}, m n_{2}, m n_{2}+q\right)$, the set of spectral numbers less than 1 is the union of the sets

$$
A_{1}=\left\{\frac{1}{n_{2}}\left(\frac{i}{n_{1}}+\frac{j}{m}\right)+\frac{r}{n_{2}} \mid 0<i<n_{1}, 0<j<m, \frac{i}{n_{1}}+\frac{j}{m}<1,0 \leq r<n_{2}\right\},
$$

$$
A_{2}=\left\{\frac{i}{n_{2}}+\frac{j}{n_{1} n_{2} m+q} \mid 0<i<n_{2}, 0<j<n_{1} n_{2} m+q, \frac{i}{n_{2}}+\frac{j}{n_{1} n_{2} m+q}<1\right\} .
$$

Let us denote by $A_{j}^{\perp}:=\left\{2-\alpha \mid \alpha \in A_{j}\right\}$, i.e. the symmetric set of $A_{j}$ with respect to 1 . Then

$$
\operatorname{Spec}(f)=A_{1} \cup A_{2} \cup A_{1}^{\perp} \cup A_{2}^{\perp} .
$$

There is a closed relationship between spectral numbers and $b$-exponents. The following result summarizes some of them which can be found for instance in [1] or [22, Remark 3.2 iii)] for (1)

Proposition 1. Let $f$ be a germ of irreducible plane curve singularity. The spectral numbers $\operatorname{Spec}(f)$ and the set $B_{f}$ of b-exponents of $f$ satisfy the following conditions:

(1) Let $\alpha_{k} \in \operatorname{Spec}(f)$, there exist a b-exponent $\beta_{k} \in B_{f}$ such that $\alpha_{k}-\beta_{k}$ is a non negative integer and $0 \leq \alpha_{k}-\beta_{k} \leq 1$.

(2) $\min B_{f}=\min \operatorname{Spec}(f)=\alpha_{1}$.

(3) $\operatorname{dim} \tilde{H}_{0}^{\prime \prime} / H_{0}^{\prime \prime}=\sum \alpha_{i}-\sum \beta_{i}$

From now on, we will study germs having a fixed characteristic sequence $C S_{n_{1}, m}^{n_{2}, q}$ satisfying (5). Our goal in this article is to show that one can compute the rational numbers that are roots of the Bernstein polynomial for any such germ. To do this we follow the same method as the one used in [7, 1,. To prove that a rational 
number is a root of the Bernstein polynomial of some function $f$, we prove that this number is a pole of some integral with a transcendental residue. We also offer algorithmic formulæ for the computation of these residues and bounds for $\operatorname{dim} \tilde{H}_{0}^{\prime \prime} / H_{0}^{\prime \prime}$.

The two main results in this paper are the following ones. We split the sets $B_{1}$ and $B_{2}$ in terms of two semigroups: $\Gamma$, the one associated with $C S_{n_{1}, m}^{n_{2}, q}$ (generated by $\left.n_{2} n_{1}, n_{2} m, n_{1} m n_{2}+q\right)$ and $\Gamma_{1}$, associated to the truncation to the first Puiseux pair (generated by $m, n_{1}$ ). Let

$$
B_{11}=\left\{\beta=\frac{m \beta_{1}+n_{1} \beta_{2}}{m n_{1} n_{2}} \in B_{1} \mid \beta_{1}, \beta_{2} \in \mathbb{Z}_{\geq 1}\right\}
$$

(which means that $k$ in (3) is in $\Gamma_{1}$ ) and

$$
B_{21}=\left\{\beta=\frac{\left(m \beta_{1}+n_{1} \beta_{2}\right) n_{2}+\left(m n_{1} n_{2}+q\right) \beta_{3}+q}{n_{2}\left(m n_{1} n_{2}+q\right)} \in B_{2} \mid \beta_{1}, \beta_{2} \in \mathbb{Z}_{\geq 1}, \beta_{3} \in \mathbb{Z}_{\geq 0}\right\} .
$$

(which means that $k$ in (41) is in $\Gamma_{2}$ ).

In Theorem 2.5 we prove that

$$
B_{11} \cup B_{21}=\bigcap_{f \in \mathcal{S}_{\mu}} R_{f, 0}
$$

where $\mathcal{S}_{\mu}$ is the set of all germs $f$ with the topological type, of the characteristic sequence $C S_{n_{1}, m}^{n_{2}, q}$ satisfying (5).

In 93 , we prove bounds for $\operatorname{dim} \tilde{H}_{0}^{\prime \prime} / H_{0}^{\prime \prime}$ for these germs. Let

$$
m=\tilde{q} n_{1}+r_{m}, 0<r_{m}<n_{1}, \quad q=h n_{2}+r_{q}, 0 \leq h, \quad 0<r_{q}<n_{2} .
$$

Then

$$
\left(n_{2}-1\right)(m-1)\left(n_{1}-1\right) \leq \operatorname{dim} \tilde{H}_{0}^{\prime \prime} / H_{0}^{\prime \prime} \leq \frac{\mu}{2}-n_{2}\left(m+n_{1}\right)-q+\tilde{q}+h+4 ;
$$

and the second inequality is generically an equality.

We end the article with several families of examples. In Theorem 4.2 it is proved that all polynomials with characteristic sequence $(4,6,6+q)$ have the same Bernstein polynomial (this is the original Yano's family). Next if a polynomial has characteristic sequence $(8,10,10+q)$ then we compute its Bernstein polynomial up to six roots (note that the Milnor number equals $63+q$ ) and we have explicit formulae to decide the remaining roots. And finally, for the last example, we find differences on the Tjurina and Bernstein stratifications. 


\section{Two VARiable integrals And Bernstein POlynomial}

Let us recall and collect some definitions, results and consequences from [1].

Definition 1.1. We say that a real polynomial $f \in \mathbb{R}[x, y]$ is positive if $f(x, y)>0$ for all $(x, y) \in[0,1]^{2}$.

Let $f \in \mathbb{R}[x, y]$ positive. Let $a_{1}, a_{2}, b_{1}, b_{2} \in \mathbb{Z}$ be fixed such that $a_{1}, a_{2} \geq$ $0, b_{1}, b_{2} \geq 1$. We denote the following complex variable integral by

$$
\mathcal{Y}(s)=\mathcal{Y}_{f, a_{1}, b_{1}, a_{2}, b_{2}}(s):=\int_{0}^{1} \int_{0}^{1} f(x, y)^{s} x^{a_{1} s+b_{1}} y^{a_{2} s+b_{2}} \frac{d x}{x} \frac{d y}{y} .
$$

Proposition 1.2 (Proposition $1.4[1]$ ). The function $\mathcal{Y}(s)$ satisfies the following properties:

(1) It is absolutely convergent for $\Re(s)>\alpha_{0}$, where $\alpha_{0}=\sup \left(-\frac{b_{1}}{a_{1}},-\frac{b_{2}}{a_{2}}\right)$

(2) It has a meromorphic continuation on $\mathbb{C}$ with poles of order at most 2 contained in $S=\left\{-\frac{b_{1}+\nu_{1}}{a_{1}}, \nu_{1} \in \mathbb{Z}_{\geq 0}\right\} \cup\left\{-\frac{b_{2}+\nu_{2}}{a_{2}}, \nu_{2} \in \mathbb{Z}_{\geq 0}\right\}$

Notation 1.3. Let $f:[0,1] \rightarrow \mathbb{R}$ be a continous function. We will denote by $G_{f}(s)$ the meromorphic continuation of

$$
\int_{0}^{1} f(t) t^{s} \frac{d t}{t}
$$

Proposition 1.4 (Proposition 1.6 [1]). With the hypotheses of Proposition 1.2 , let $\nu_{1} \in \mathbb{Z}_{\geq 0}$ be fixed and such that $\alpha=-\frac{b_{1}+\nu_{1}}{a_{1}} \neq-\frac{b_{2}+\nu_{2}}{a_{2}}$ for all $\nu_{2} \in \mathbb{Z}_{\geq 0}$, then the pole of $\mathcal{Y}(s)$ at $\alpha$ is simple and

$$
\operatorname{Res}_{s=\alpha} \mathcal{Y}(s)=\frac{1}{\nu_{1} ! a_{1}} G_{h_{\nu_{1}, \alpha, x}}\left(a_{2} \alpha+b_{2}\right), \quad h_{\nu_{1}, \alpha, x}(y):=\frac{\partial^{\nu_{1}} f^{\alpha}}{\partial x^{\nu_{1}}}(0, y) .
$$

Note that, under the hypotheses of the above Proposition, $G_{h_{\nu_{1}, \alpha, x}}\left(a_{2} s+b_{2}\right)$ admits an integral expression which is absolutely convergent and holomorphic for $\Re(s)>-N_{2}-1$, with $N_{2}$ such that $\alpha>-\frac{b_{2}+N_{2}+1}{a_{2}}$, see the proof in [1] of the above Proposition 1.2 .

We collect next a result which relates these integrals with the beta function $\boldsymbol{B}\left(s_{1}, s_{2}\right)$.

Lemma 1.5 (Lemma $1.8[1]$ ). Let $p \in \mathbb{N}$ and $c \in \mathbb{R}_{>0}$. Given $s_{1}, s_{2} \in \mathbb{C}$ such that $-\alpha=s_{1}+s_{2}>0$ then

$$
G_{\left(y^{p}+c\right)^{\alpha}}\left(p s_{1}\right)+G_{\left(1+c x^{p}\right)^{\alpha}}\left(p s_{2}\right)=\frac{c^{-s_{2}}}{p} \boldsymbol{B}\left(s_{1}, s_{2}\right)
$$

where $\boldsymbol{B}$ is the beta function. 
Our goal in this article is to show that one can compute the rational numbers that are roots of the Bernstein polynomial for any function having characteristic sequence $C S_{n_{1}, m}^{n_{2}, q}$ satisfying (5). To do this we follow the same methods and ideas as the one used by Pi. Cassou-Noguès in [5, 4, 6, 17, 1,. To prove that a rational number is a root of the Bernstein polynomial of some function $f$, we prove that such a number is pole of some integral whose residue is a transcendental number.

To use the method one needs to start with a real polynomial $f \in \mathbb{R}[x, y]$ whose complex analytic germ at the origin has $C S_{n_{1}, m}^{n_{2}, q}$ as characteristic sequence.

Definition 1.6. A polynomial $f \in \mathbb{R}[x, y]$ is said to be of type $\left(n_{1} n_{2}, m n_{2}, m n_{2}+\right.$ $q)^{+}$if it satisfies:

$$
f(x, y)=\left(x^{n_{1}}+y^{m}+h_{1}(x, y)\right)^{n_{2}}+x^{a} y^{b}+h_{2}(x, y)
$$

where

$\left(\mathrm{G}^{+} 1\right) h_{1}(x, y)=\sum_{(i, j) \in \mathcal{P}_{n_{1}, m}} a_{i j} x^{i} y^{j} \in \mathbb{R}[x, y]$, where

$$
\mathcal{P}_{n_{1}, m}:=\left\{(i, j) \in \mathbb{Z}_{\geq 0}^{2} \mid m i+n_{1} j>m n_{1}\right\}
$$

$\left(\mathrm{G}^{+} 2\right) a, b \geq 0$ such that $a m+b n_{1}=m n_{1} n_{2}+q$;

$\left(\mathrm{G}^{+} 3\right)$ the polynomial $h_{2} \in \mathbb{R}[x, y]$, whose support is disjoint from the other terms of $f$, satisfies that the characteristic sequence of $f$ is $C S_{n_{1}, m}^{n_{2}, q}$.

Proposition 1.7. Let $f \in \mathbb{R}[x, y]$ be a real polynomial as in (1.4) satisfying $\left(\left(\mathrm{G}^{+} 1\right)\right]$, $\left(\mathrm{G}^{+} 2\right)$ and $\left(\mathrm{G}^{+} 3\right)$. Then there exists a domain $D=[0, \eta]^{2}$, with $\eta \leq 1$, such that $f>0$ in $[0, \eta]^{2} \backslash\{(0,0)\}$.

Proof. Note that the real zero locus of $x^{n_{1}}+y^{m}$ intersects $[0,1]^{2}$ only at $(0,0)$. Since the real zero locus of $f=0$ is a deformation of the previous one, then there is $\eta>0$ for which the statement follows.

For $\beta_{1}, \beta_{2} \in \mathbb{Z}_{\geq 1}$, and $f$ of type $\left(n_{1} n_{2}, m n_{2}, m n_{2}+q\right)^{+}$one defines:

$$
I_{+}\left(f, \beta_{1}, \beta_{2}\right)(s):=\int_{0}^{1} \int_{0}^{1} f(x, y)^{s} x^{\beta_{1}} y^{\beta_{2}} \frac{d x}{x} \frac{d y}{y} .
$$

Proposition $1.8([1])$. Let $f$ be of type $\left(n_{1} n_{2}, m n_{2}, m n_{2}+q\right)^{+}$and $\beta_{1}, \beta_{2} \in \mathbb{Z}_{\geq 1}$. Then the integral $I_{+}\left(f, \beta_{1}, \beta_{2}\right)(s)$ is absolutely convergent for $\Re(s)>-\frac{\beta_{1} m+\beta_{2} n_{1}}{m n_{1} n_{2}}$ and may have simple poles only for $s=-\frac{\beta_{1} m+\beta_{2} n_{1}+\nu}{m n_{1} n_{2}}, \nu \in \mathbb{Z}_{\geq 0}$.

Next we show the algorithmic description of [1, Section 3] to compute the residue of the corresponding family of poles. Let us see show to compute the residue at the eventual pole $\alpha=-\frac{\beta_{1} m+\beta_{2} n_{1}+\nu}{m n_{1} n_{2}}$ of the integral $I_{+}\left(f, \beta_{1}, \beta_{2}\right)(s)$. Let

$$
\tilde{f}(x, y)=f\left(x^{m}, y^{n_{1}}\right)
$$


and let $f_{1}$ and $f_{2}$ be defined by

$$
\tilde{f}(x, x y)=x^{n_{1} n_{2} m} f_{1}(x, y), \quad \tilde{f}(x y, y)=y^{n_{1} n_{2} m} f_{2}(x, y) .
$$

Thus the residue of $\alpha=-\frac{\beta_{1} m+\beta_{2} n_{1}+\nu}{m n_{1} n_{2}}$ of the integral $I_{+}\left(f, \beta_{1}, \beta_{2}\right)(s)$ equals

$$
\operatorname{Res}_{s=\alpha} I_{+}\left(f, \beta_{1}, \beta_{2}\right)(s)=\frac{1}{\nu ! m n_{1} n_{2}}\left(G_{h_{\nu, \alpha, x}^{1}}\left(n_{1} \beta_{2}\right)+G_{h_{\nu, \alpha, y}^{2}}\left(m \beta_{1}\right)\right) ;
$$

where

$$
h_{\nu, \alpha, x}^{1}(y)=\frac{\partial^{\nu} f_{1}^{\alpha}}{\partial x^{\nu}}(0, y), \quad \text { and } \quad h_{\nu, \alpha, y}^{2}(x)=\frac{\partial^{\nu} f_{2}^{\alpha}}{\partial y^{\nu}}(x, 0) .
$$

We define now a simplified version of polynomials of type $\left(n_{1} n_{2}, m n_{2}, m n_{2}+q\right)^{-}$ defined in [1].

Definition 1.9. A polynomial $f \in \mathbb{R}[x, y]$ is said to be of type $\left(n_{1} n_{2}, m n_{2}, m n_{2}+\right.$ $q)_{s}^{-}$if it satisfies:

$$
f(x, y)=g(x, y)^{n_{2}}+x^{a} y^{b}+h_{2}(x, y)
$$

where $g(x, y):=x^{n_{1}}-y^{m}$

$\left(\mathrm{G}^{-} 1\right) a, b \geq 0$ are as in $\left(\mathrm{G}^{+} 2\right)$.

$\left(\mathrm{G}^{-} 2\right)$ The polynomial $h_{2} \in \mathbb{R}[x, y]$, whose support is disjoint from the first terms, satisfies that the characteristic sequence of $f$ is $C S_{n_{1}, m}^{n_{2}, q}$.

$\left(\mathrm{G}^{-} 3\right)$ There is an $\epsilon>0$ such that for $\mathcal{D}:=\left\{(x, y) \in \mathbb{R}^{2} \mid 0 \leq x \leq \epsilon, 0 \leq y \leq\right.$ $\left.x^{\frac{n_{1}}{m}}\right\}$, we have that $f>0$ on $\mathcal{D} \backslash\{(0,0)\}$.

Proposition 1.10. For each $f$ as in (1.7) satisfying the conditions $\left(\mathrm{G}^{-} 1\right)$, and $\left(\mathrm{G}^{-2}\right)$ then there is $\epsilon>0$ and a domain $\mathcal{D}:=\left\{(x, y) \in \mathbb{R}^{2} \mid 0 \leq x \leq \epsilon, 0 \leq y \leq\right.$ $\left.x^{\frac{n_{1}}{m}}\right\}$ for which $f$ satisfies $\left(\mathrm{G}^{-} 3\right)$ in $D$, that is $f$ is of type $\left(n_{1} n_{2}, m n_{2}, m n_{2}+q\right)_{s}^{-}$.

Proof. It is enough to take a suitable truncation of a Puiseux expansion of $f$ (which has no term between the two characteristic terms).

For $\beta_{1}, \beta_{2} \in \mathbb{Z}_{\geq 1}, \beta_{3} \in \mathbb{Z}_{\geq 0}$ and $f$ of type $\left(n_{1} n_{2}, m n_{2}, m n_{2}+q\right)_{s}^{-}$we set:

$$
\mathcal{I}_{-}\left(f, \beta_{1}, \beta_{2}, \beta_{3}\right)(s):=\iint_{\mathcal{D}} f(x, y)^{s} x^{\beta_{1}} y^{\beta_{2}} g(x, y)^{\beta_{3}} \frac{d x}{x} \frac{d y}{y} .
$$

Proposition $1.11([1])$. Let $f \in \mathbb{R}[x, y]$ be a polynomial of type $\left(n_{1} n_{2}, m n_{2}, m n_{2}+\right.$ $q)_{s}^{-}, \beta_{1}, \beta_{2} \in \mathbb{Z}_{\geq 1}$ and $\beta_{3} \in \mathbb{Z}_{\geq 0}$. Then the integral $\mathcal{I}_{-}\left(f, \beta_{1}, \beta_{2}, \beta_{3}\right)(s)$ is convergent for $\Re(s)>-\frac{\beta_{1} m+\beta_{2} n_{1}+\beta_{3} m n_{1}}{m n_{1} n_{2}}$ and its set of poles is contained in the set

$$
P_{1} \cup \bigcup_{i \in \mathbb{Z} \geq 1} P_{2, i}
$$


where

$$
P_{1}:=\left\{-\frac{m \beta_{1}+n_{1} \beta_{2}+m n_{1} \beta_{3}+\nu}{m n_{1} n_{2}} \mid \nu \in \mathbb{Z}_{\geq 0}\right\}
$$

and

$$
P_{2, i}:=\left\{-\frac{n_{2}\left(m \beta_{1}+n_{1} \beta_{2}+m n_{1} \beta_{3}+\right)+q\left(\beta_{3}+i\right)+\nu}{n_{2}\left(m n_{1} n_{2}+q\right)} \mid \nu \in \mathbb{Z}_{\geq 0}\right\}
$$

The poles have at most order two. The poles may have order two at the values contained in $P_{1}$ and $P_{2, i}$ for some $i$.

We shall give the residues at the eventual simple poles in $P_{2, i}$. Let $\tilde{f}, \tilde{\tilde{f}}, \hat{f}$ be defined by

$$
f\left(x^{m}, y^{n_{1}}\right)=\tilde{f}(x, y), \quad \tilde{f}(x, x y)=x^{m n_{1} n_{2}} \tilde{\tilde{f}}(x, y), \quad \hat{f}(x, y)=\tilde{\tilde{f}}(x, 1-y) .
$$

Let $\hat{\hat{f}}, f_{1}, f_{2}$ be defined by

$$
\hat{f}\left(x^{n_{2}}, y^{q}\right)=\hat{\hat{f}}(x, y), \quad \hat{\hat{f}}(x, x y)=x^{n_{2} q} f_{1}(x, y), \quad \hat{\hat{f}}(x y, y)=y^{n_{2} q} f_{2}(x, y) .
$$

Let us denote

$$
g\left(x^{m}, y^{n_{1}}\right)=\tilde{g}_{Y}(x, y)=x^{m n_{1}}-y^{m n_{1}}, \quad \tilde{\tilde{g}}(y)=\frac{\tilde{g}(x, x y)}{x^{m n_{1}}}=1-y^{m n_{1}} .
$$

In particular,

$$
\tilde{\tilde{g}}(1-y)=y Q(y), \quad Q(0)=n_{1} .
$$

Let us define

$$
\tilde{Q}(y)=Q(y)^{\beta_{3}}(1-y)^{n_{1} \beta_{2}-1}, \quad \tilde{Q}(y)=\sum b_{i} y^{i-1} .
$$

Thus the integral $\mathcal{I}_{-}\left(f, \beta_{1}, \beta_{2}, \beta_{3}\right)(s)$ has residue for

$$
s=\alpha=-\frac{n_{2}\left(m \beta_{1}+n_{1} \beta_{2}+m n_{1} \beta_{3}\right)+q\left(\beta_{3}+i\right)+\nu}{n_{2}\left(m n_{1} n_{2}+q\right)}
$$

equals

$$
\begin{gathered}
\operatorname{Res}_{s=\alpha} \mathcal{I}_{-}\left(f, \beta_{1}, \beta_{2}, \beta_{3}\right)(s)=\frac{1}{n_{2} q} \sum_{i, \nu} \frac{1}{\nu !} b_{i}\left(G_{h_{\nu, \alpha, x}^{1}}\left(q\left(\beta_{3}+i\right)\right)+\right. \\
G_{h_{\nu, \alpha, y}^{2}}\left(n_{2}\left(m n_{1} n_{2} \alpha+m \beta_{1}+n_{1} \beta_{2}+m n_{1} \beta_{3}\right)\right)
\end{gathered}
$$

where

$$
h_{\nu, \alpha, x}^{1}(y)=\frac{\partial^{\nu} f_{1}^{\alpha}}{\partial x^{\nu}}(0, y), \quad \text { and } \quad h_{\nu, \alpha, y}^{2}(x)=\frac{\partial^{\nu} f_{2}^{\alpha}}{\partial y^{\nu}}(x, 0) ;
$$

recall also that $G_{f}(s)$ is the meromorphic continuation of $\int_{0}^{1} f(t) t^{s} \frac{d t}{t}$.

Remark 1.12. We may assume $\epsilon=1$ after a suitable change of variables. 
Let us summarize the links between these integrals and the Bernstein polynomial. We are using ideas in [4, 5, 6, 1]. Let us fix notations that may cover both cases. We fix $f$ with the following properties:

(B1) The characteristic sequence of $f \in \mathbb{R}[x, y]$ is $C S_{n_{1}, m}^{n_{2}, q}$.

(B2) The polynomial $Y\left(x^{\frac{1}{m}}\right) \in \mathbb{R}\left[x^{\frac{1}{m}}\right]$ is either 1 (for the +-case) or $x^{\frac{n_{1}}{m}}$ for the $-{ }$-case

(B3) $\mathcal{D}:=\left\{(x, y) \in \mathbb{R}^{2} \mid 0 \leq x \leq 1,0 \leq y \leq Y\left(x^{\frac{1}{m}}\right)\right\}, g(x, y)=x^{n_{1}} \pm y^{m}$.

(B4) $f(x, y)>0 \forall(x, y) \in \mathcal{D} \backslash\{(0,0)\}$.

Let $\beta_{1}, \beta_{2} \in \mathbb{Z}_{\geq 1}$ and $\beta_{3} \in \mathbb{Z}_{\geq 0}$ (equals 0 for the + -case). Let us consider the integral

$$
\mathcal{I}_{ \pm}\left(f, \beta_{1}, \beta_{2}, \beta_{3}\right)(s):=\iint_{\mathcal{D}} f(x, y)^{s} x^{\beta_{1}} y^{\beta_{2}} g(x, y)^{\beta_{3}} \frac{d x}{x} \frac{d y}{y} .
$$

Theorem 1.13 ([1, Theorem 5.3]). Let $f(x, y) \in \mathbb{K}[x, y]$ be a polynomial defining an irreducible germ of complex plane curve at the origin which has two Puiseux pairs and its algebraic monodromy has distinct eigenvalues and such that $\mathbb{K}$ is an algebraic extension of $\mathbb{Q}$. Let $\alpha$ be a pole of $\mathcal{I}_{ \pm}\left(f, \beta_{1}, \beta_{2}, \beta_{3}\right)(s)$ with transcendental residue, and such that $\alpha+1$ is not a pole of $\mathcal{I}_{ \pm}\left(f, \beta_{1}^{\prime}, \beta_{2}^{\prime}, \beta_{3}^{\prime}\right)(s)$ for any $\left(\beta_{1}^{\prime}, \beta_{2}^{\prime}, \beta_{3}^{\prime}\right)$. Then $\alpha$ is root of the Bernstein-Sato polynomial $b_{f}(s)$ of $f$.

\section{Determination of the Set of COMmon roots of the $\mu$-Constant STRATUM}

Let $f$ be an irreducible germ of plane curve whose characteristic sequence is $C S_{n_{1}, m}^{n_{2}, q}$ satisfying (5). The Bernstein-Sato polynomial of a germ $f$ with this characteristic sequence, depends on $f$, but there is a generic Bernstein polynomial $b_{\mu \text {,gen }}(s)$ : for every $\mu$-constant deformation of such an $f$, there is a Zariski dense open set $\mathcal{U}$ on which the Bernstein-Sato polynomial of any germ in $\mathcal{U}$ equals $b_{\mu, g e n}(s)$.

Proposition 2.1 ([27, Corollary 21]). Let $f_{t}(x)$ be a $\mu$-constant analytic deformation of an isolated hypersurface singularity $f_{0}(x)$. If all eigenvalues of the monodromy are pairwise different, then all roots of the reduced Bernstein-Sato polynomial $\tilde{b}_{f_{t}}(s)$ depend lower semi-continously upon the parameter $t$.

Proposition 2.2 ([11, Corollary 5.1],[10]). Let $f(x)$ be a germ of an isolated hypersurface singularity. Then for each spectral number $\alpha \in \operatorname{Spec}(f)$ such that $\alpha<\alpha_{1}+1$, then $-\alpha$ is root of the Bernstein polynomial $b_{f}(s)$. 
Consequently, for a $\mu$-constant analytic deformation $f_{t}(x)$ of an isolated hypersurface singularity germ $f_{0}(x)$, for every $\alpha$ in

$$
\mathcal{E}:=\left\{\alpha: \alpha \in \operatorname{Spec}(f) \text { and } \alpha<\alpha_{1}+1\right\}
$$

then $-\alpha$ is root of every Bernstein polynomial $b_{f_{t}}(s)$ for every $t$.

Remark 2.3. Note that we follow Saito's convention for the exponents and the spectral numbers, which differs by 1 from the convention in [11].

The following Corollary is a consequence of Proposition 2.1.

Corollary 2.4. Let $f_{0}(x, y)$ be an irreducible germ of plane curve whose monodromy has distinct eigenvalues. Let $-\alpha$ be a root of the local Bernstein-Sato polynomial $b_{f_{0}}(s)$. Then, either $-\alpha$ or $-(\alpha+1)$ is a root of $b_{\mu, g e n}(s)$.

Let $S_{\mu}$ be the (non-singular) $\mu$-constant stratum of $f$ at 0 . Let $R_{f}$ be the set of the roots of $b_{f}(-s)$. For every $g \in S_{\mu}$ and since $g$ has isolated singularities then

$$
R_{g} \subset \operatorname{Spec}(g) \cup\{\alpha-1 \mid \alpha \in \operatorname{Spec}(g)\} \text {, see [10]. }
$$

Since the spectral numbers are constant in a $\mu$-constant deformation then, in the image of the map $S_{\mu} \rightarrow \mathbb{C}[s]: g \mapsto b_{g}(s)$ there are finitely many polynomials.

The aim of this section is to describe the set of common roots of the Bernstein polynomials of the $\mu$-constant stratum, that is, the set

$$
\mathcal{C} R_{\mu}:=\bigcap_{f \in S_{\mu}} R_{f}
$$

By Proposition (2.2) the set $\mathcal{E} \subset \mathcal{C} R_{\mu}$.

In [1], we proved that the set of roots of the Bernstein polynomial $b_{\mu, \text { gen }}(-s)$ is $B_{1} \cup B_{2}$. We split these sets $B_{1}$ and $B_{2}$ using (9) and (10) and we set $B_{12}=$ $B_{1} \backslash B_{11}, B_{22}=B_{2} \backslash B_{21}$.

The aim of this part is to prove

Theorem 2.5. Let $\mathcal{C} R_{\mu}$ be the set of common roots of the Bernstein polynomials of every irreducible germ of plane curve whose characteristic sequence is $C S_{n_{1}, m}^{n_{2}, q}$ satisfying (5). Then $\mathcal{C} R_{\mu}=B_{11} \cup B_{21}$.

We divide the proof in three parts.

Proposition 2.6. $B_{11} \cup B_{21} \subset \mathcal{C} R_{\mu}$.

Proof. Let $\alpha \in B_{11} \cup B_{21} \subset B_{1} \cup B_{2}$ and let $f$ be a fixed germ in $S_{\mu}$. As $-\alpha$ is a root of the generic Bernstein polynomial, if $-\alpha$ is not a root of the Bernstein polynomial of $f$, then $-(\alpha+1)$ is by Corollary 2.4. Then $-\alpha-1>-2$ and $\alpha<1$. 
In particular, if $\alpha>1$ then $\alpha$ is a root for any germ. We need only to study $B^{\prime}=\left(B_{11} \cup B_{21}\right) \cap\{\alpha \in \mathbb{Q} \mid \alpha<1\}$. Hence, we need only to prove that $B^{\prime} \subset \mathcal{C} R_{\mu}$.

To do this, we use a result of B. Lichtin ([15, Section 3, Corollary 2]) and Loeser ([16, Remarque III.3.5]). Let us state it.

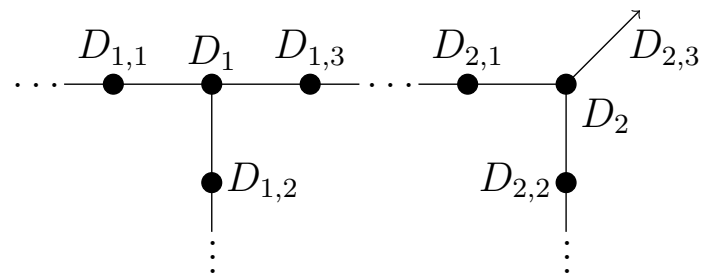

FiguRE 1. Resolution graph

Let us consider an embedded resolution of $f$, see Figure 1, together with a 2differential form $\omega$. For a divisor $D_{j}$, let us denote $N_{j}:=\operatorname{ord}_{D_{j}}(f)$ and $\nu_{j}(\omega):=$ $\operatorname{ord}_{D_{j}}(\omega)+1$.

In the resolution of $f$ we have two branching divisors that we denote by $D_{1}, D_{2}$. We denote by $D_{j, 1}, D_{j, 2}, D_{j, 3}$ the divisors adjacent to $D_{j}, j=1,2$. For $j=1,2, k=$ $1,2,3$ we set

$$
\epsilon_{j, k}(\omega):=\frac{1}{N_{j}} \operatorname{det}\left(\begin{array}{cc}
N_{j} & N_{j, k} \\
\nu_{j}(\omega) & \nu_{j, k}(\omega)
\end{array}\right) \stackrel{\bmod \mathbb{Z}}{\equiv}-\frac{\nu_{j}(\omega) N_{j, k}}{N_{j}} .
$$

If the following conditions hold:

- $\epsilon_{j, k}(\omega)$ is not an integer for $k=1,2,3$,

- $\frac{\nu_{j}(\omega)}{N_{j}}<1$;

then $-\frac{\nu_{j}(\omega)}{N_{j}}$ is a root of the Bernstein polynomial of $f$. We have the relation $\sum_{k} \epsilon_{j, k}(\omega)=-2$ for $j=1,2$.

It is easily checked that

$$
N_{1}=m n_{1} n_{2}, \quad N_{2}=n_{2}\left(m n_{1} n_{2}+q\right),
$$

and

$$
N_{1,1}=n_{1} n_{2} \alpha_{1,1}, \quad N_{1,2}=m n_{2} \alpha_{1,2}, \quad N_{1,3}=\left(m n_{1} \alpha_{1,3}^{\prime}+1\right) n_{2},
$$

where

$$
\alpha_{1,1} n_{1}+1=\alpha_{1,1}^{\prime} m, \quad \alpha_{1,2} m+1=\alpha_{1,2}^{\prime} n_{1}, \quad \alpha_{1,3}+n_{2}=\alpha_{1,3}^{\prime} q,
$$

all positive integers. We also have

$$
N_{2,1}=n_{2}\left(m n_{1} \alpha_{2,1}^{\prime}+\alpha_{2,1}\right), \quad N_{2,2}=\alpha_{2,2}\left(m n_{1} n_{2}+q\right), \quad N_{2,3}=1,
$$


where

$$
\alpha_{2,1} n_{2}+1=\alpha_{2,1}^{\prime} q, \quad \alpha_{2,2} q+1=\alpha_{2,2}^{\prime} n_{2},
$$

again all positive integers.

Let us check the conditions for $\alpha=\frac{m \beta_{1}+n_{1} \beta_{2}}{m n_{1} n_{2}} \in B_{11} \cap B^{\prime}$ with the form $\omega_{1}=$ $x^{\beta_{1}-1} y^{\beta_{2}-1} d x d y$. Since $\nu_{1}\left(\omega_{1}\right)=m \beta_{1}+n_{1} \beta_{2}$, we have that $\alpha=\frac{\nu_{1}\left(\omega_{1}\right)}{N_{1}}$. Only the non-integer condition must be checked. We have:

$$
\begin{aligned}
& -\epsilon_{1,1}\left(\omega_{1}\right) \stackrel{\bmod \mathbb{Z}}{\equiv} \frac{\left(m \beta_{1}+n_{1} \beta_{2}\right) \alpha_{1,1} n_{1} n_{2}}{m n_{1} n_{2}} \stackrel{\bmod \mathbb{Z}}{\equiv} \frac{n_{1} \beta_{2} \alpha_{1,1}}{m} \stackrel{\bmod \mathbb{Z}}{\equiv}-\frac{\beta_{2}}{m} \notin \mathbb{Z} \\
& -\epsilon_{1,2}\left(\omega_{1}\right) \stackrel{\bmod \mathbb{Z}}{\equiv} \frac{\left(m \beta_{1}+n_{1} \beta_{2}\right) \alpha_{2,1} m n_{2}}{m n_{1} n_{2}} \stackrel{\bmod \mathbb{Z}}{\equiv} \frac{m \beta_{1} \alpha_{1,2}}{n_{1}} \stackrel{\bmod \mathbb{Z}}{\equiv}-\frac{\beta_{1}}{n_{1}} \notin \mathbb{Z} \\
& -\epsilon_{1,3}\left(\omega_{1}\right) \stackrel{\bmod \mathbb{Z}}{\equiv} \frac{\left(m \beta_{1}+n_{1} \beta_{2}\right)\left(m n_{1} \alpha_{1,3}^{\prime}+1\right) n_{2}}{m n_{1} n_{2}} \stackrel{\bmod \mathbb{Z}}{\equiv} \frac{m \beta_{1}+n_{1} \beta_{2}}{m n_{1}} \notin \mathbb{Z}
\end{aligned}
$$

Finally we check the conditions for $\alpha=\frac{\left(m \beta_{1}+n_{1} \beta_{2}\right) n_{2}+q+\left(m n_{1} n_{2}+q\right) \beta_{3}}{n_{2}\left(m n_{1} n_{2}+q\right)} \in B_{21} \cap B^{\prime}$ with the form $\omega_{2}=x^{\beta_{1}-1} y^{\beta_{2}-1} g_{Y}(x, y)^{\beta_{3}} d x d y$. Since $\nu_{2}\left(\omega_{2}\right)=\left(m \beta_{1}+n_{1} \beta_{2}\right) n_{2}+$ $q+\left(m n_{1} n_{2}+q\right) \beta_{3}$, we have that $\alpha=\frac{\nu_{2}\left(\omega_{2}\right)}{N_{2}}$. Let us check the non-integer condition. We have:

$$
\begin{gathered}
-\epsilon_{2,1}\left(\omega_{2}\right) \stackrel{\bmod \mathbb{Z}}{\equiv} \frac{\left(\left(m \beta_{1}+n_{1} \beta_{2}\right) n_{2}+q+\left(m n_{1} n_{2}+q\right) \beta_{3}\right)\left(\alpha_{2,1}^{\prime} m n_{1}+\alpha_{2,1}\right) n_{2}}{n_{2}\left(m n_{1} n_{2}+q\right)} \stackrel{\bmod \mathbb{Z}}{-\frac{m \beta_{1}+n_{1} \beta_{2}-m n_{1}}{m n_{1} n_{2}+q}} \\
\epsilon_{2,2}\left(\omega_{2}\right) \stackrel{\bmod \mathbb{Z}}{\equiv} \frac{\left(\left(m \beta_{1}+n_{1} \beta_{2}\right) n_{2}+q+\left(m n_{1} n_{2}+q\right) \beta_{3}\right) \alpha_{2,2}\left(m n_{1} n_{2}+q\right)}{n_{2}\left(m n_{1} n_{2}+q\right)} \stackrel{\bmod }{\equiv} \frac{\mathbb{Z} \beta_{3}+1}{n_{2}} \\
\epsilon_{2,3}\left(\omega_{2}\right) \stackrel{\bmod \mathbb{Z}}{\equiv} \frac{\left(\left(m \beta_{1}+n_{1} \beta_{2}\right) n_{2}+q+\left(m n_{1} n_{2}+q\right) \beta_{3}\right)}{n_{2}\left(m n_{1} n_{2}+q\right)} .
\end{gathered}
$$

None of the above numbers is an integer.

Proposition 2.7. For all $\beta \in B_{12}$, there exits $f_{\beta}$ with characteristic sequence $\left(n_{1} n_{2}, m n_{2}, m n_{2}+q\right)$ such that $-\beta$ is not a root of the Bernstein polynomial of $f_{\beta}$.

Proof. Let $\beta \in B_{12}$, i.e. there exists $k \geq 1$ such that $\beta=\frac{n_{1}+m+k}{n_{1} n_{2} m}$ and $k$ is not in the semigroup $\Gamma_{1}$ generated by $m, n_{1}$. Then $\beta+1=\frac{n_{1}+m+n_{1} m m_{2}+k}{n_{1} n_{2} m}$. Since the conductor of $\Gamma_{1}$ is $m n_{1}-m-n_{1}$, then $n_{1}+m+n_{1} m m_{2}+k \in \Gamma_{1}$ and there exist $\beta_{1}$ and $\beta_{2}$ such that $1+\beta=\frac{m \beta_{1}+n_{1} \beta_{2}}{m n_{1} n_{2}}$. In particular, if $f$ is of type $\left(n_{1} n_{2}, m n_{2}, m n_{2}+q\right)^{+}$, then

$$
\operatorname{Res}_{s=-\beta-1} I\left(f, \beta_{1}, \beta_{2}\right)(s) \neq 0
$$

is transcendental, see [1, Proposition 3.3]. 
Claim 2.8. There exists $f$ of type $\left(n_{1} n_{2}, m n_{2}, m n_{2}+q\right)^{+}$such that

$$
\operatorname{Res}_{s=-\beta} I_{+}\left(f, \beta_{1}, \beta_{2}\right)(s)=0, \quad \forall\left(\beta_{1}, \beta_{2}\right) \in \mathbb{Z}_{\geq 1}
$$

Assume that Claim 2.8 has been proved (see the Appendix A). For such an $f$, $-\beta-1$ is a root of the Bernstein polynomial of $f$. Since the hypotheses of Theorem 1.13 hold (see also [1, Theorem 5.3]) $-\beta$ is not such a root since the monodromy has distinct eigenvalues. Then there exists $f$ such that $-\beta$ is not root of the Bernstein polynomial $b_{f, 0}(s)$.

Proposition 2.9. For all $\beta \in B_{22}$, there exists $f_{\beta}$ with characteristic sequence $\left(n_{1} n_{2}, m n_{2}, m n_{2}+q\right)$ such that $-\beta$ is not a root of the Bernstein polynomial of $f_{\beta}$.

Proof. Let $\beta \in B_{22}$, i.e we have $\beta=\frac{n_{2}\left(m+n_{1}\right)+q+k}{n_{2}\left(m n_{1} n_{2}+q\right)}$ and $k \notin \Gamma$, where $\Gamma$ is the semigroup generated by $m n_{2}, n_{1} n_{2}$ and $m n_{1} n_{2}+q$; its conductor is

$$
n_{2}\left(m n_{1} n_{2}+q\right)-\left(m+n_{1}\right) n_{2}-q+1
$$

In particular, $n_{2}\left(m n_{1} n_{2}+q\right)+k \in \Gamma$ and there exist $\beta_{1}, \beta_{2}, \beta_{3}$ such that

$$
1+\beta=\frac{m \beta_{1}+n_{1} \beta_{2}+\left(m n_{1} n_{2}+q\right) \beta_{3}}{n_{2}\left(m n_{1} n_{2}+q\right)} .
$$

As a consequence, for any $f$ of type $\left(n_{1} n_{2}, m n_{2}, m n_{2}+q\right)^{-}$, we have that

$$
\underset{s=-\beta-1}{\operatorname{Res}} I_{-}\left(f, \beta_{1}, \beta_{2}, \beta_{3}\right)(s) \neq 0
$$

is transcendental, see [1, Proposition 4.3].

Claim 2.10. There exists $f$ of type $\left(n_{1} n_{2}, m n_{2}, m n_{2}+q\right)^{-}$such that

$$
\operatorname{Res}_{s=-\beta} I_{-}\left(f, \beta_{1}, \beta_{2}, \beta_{3}\right)(s)=0, \quad \forall\left(\beta_{1}, \beta_{2}\right) \in \mathbb{Z}_{\geq 1}, \beta_{3} \in \mathbb{Z}_{\geq 0} .
$$

Assuming this Claim (to be proved in the Appendix A), the result follows the arguments of the end of the proof of Proposition 2.7 .

\section{Bounds FOR $\operatorname{dim} \tilde{H}_{0}^{\prime \prime} / H_{0}^{\prime \prime}$}

Proposition 3.1. Let $f$ be an irreducible germ of plane curve whose characteristic sequence is $C S_{n_{1}, m}^{n_{2}, q}$ satisfying (5). Define the integers $\tilde{q}$ and $h$ by $m=\tilde{q} n_{1}+r_{m}, 0<$ $r_{m}<n_{1}, q=h n_{2}+r_{q}, 0 \leq h, 0<r_{q}<n_{2}$. Then

$$
\operatorname{dim} \tilde{H}_{0}^{\prime \prime} / H_{0}^{\prime \prime} \leq \frac{\mu}{2}-n_{2}\left(m+n_{1}\right)-q+\tilde{q}+h+4
$$

and generically $\operatorname{dim} \tilde{H}_{0}^{\prime \prime} / H_{0}^{\prime \prime}=\frac{\mu}{2}-n_{2}\left(m+n_{1}\right)-q+\tilde{q}+h+4$ 
Proof. One can compute the dimension $\operatorname{dim} \tilde{H}_{0}^{\prime \prime} / H_{0}^{\prime \prime}$ using Proposition 1, that is $\operatorname{dim} \tilde{H}_{0}^{\prime \prime} / H_{0}^{\prime \prime}=\sum_{i=1}^{\mu} \alpha_{i}-\sum_{i=1}^{\mu} \beta_{i}$, the $\alpha_{i}$ being the spectral numbers, which are constant in the $\mu$-constant stratum $S_{\mu}$, and the $\beta_{i}$ being the $b$-exponents of $f$, which, under the conditions of the Proposition, equal the roots of the local Bernstein-Sato polynomial $b_{f}(-s)$.

The fact that the upper bound of $\operatorname{dim} \tilde{H}_{0}^{\prime \prime} / H_{0}^{\prime \prime}$ is attained generically is a consequence of the proof of Yano's conjecture in [1], see also Proposition 2.1, so that the roots of $b_{\mu, g e n}(-s)$ is the set $B_{1} \cup B_{2}$.

We start by adding the spectral numbers $\alpha_{i} \in \operatorname{Spec}(f)=A_{1} \cup A_{1}^{\perp} \cup A_{2} \cup A_{2}^{\perp}$, see (88). Using the symmetry of the spectral numbers, i.e. $\alpha_{i}+\alpha_{\mu-(i-1)}=2$, for each characteristic pair, $k=1,2$, one has:

$$
\sum_{\alpha_{i} \in A_{k} \cup A_{k}^{\perp}} \alpha_{i}=2\left|A_{k}\right|
$$

Using Saito's result [23],

$$
\left.\left|A_{1}\right|=\frac{n_{2}(m-1)\left(n_{1}-1\right)}{2} \quad \text { (6) }\right), \quad\left|A_{2}\right|=\frac{\left(n_{2}-1\right)\left(n_{1} n_{2} m+q-1\right)}{2},
$$

Next we compute the sum of the roots of $b_{\mu, g e n}(-s)$ which is the same as the sum of the elements of $B_{1} \cup B_{2}$. Let us start with

$$
B_{1}:=\left\{\sigma=\frac{m+n_{1}+k}{m n_{1} n_{2}}: 0 \leq k<m n_{1} n_{2}, \text { and } n_{2} m \sigma, n_{2} n_{1} \sigma \notin \mathbb{Z}\right\} .
$$

Since $\operatorname{gcd}\left(n_{1}, m\right)=1$ and define $N_{1}:=m n_{1} n_{2}$ and for the first characteristic exponent one has

$$
\begin{gathered}
\sum_{\beta_{i} \in B_{1}} \beta_{i}=\sum_{k=0}^{m n_{1} n_{2}-1} \frac{m+n_{1}+k}{m n_{1} n_{2}}-\sum_{\substack{0 \leq k<m n_{1} n_{2} \\
n_{1}+k \in m \mathbb{Z}}} \frac{m+n_{1}+k}{m n_{1} n_{2}} \\
-\sum_{\substack{0 \leq k<m n_{1} n_{2} \\
m+k \in n_{1} \mathbb{Z}}} \frac{m+n_{1}+k}{N_{1}}+\sum_{\substack{0 \leq k<m n_{1} n_{2} \\
n_{1}+m+k \in m n_{1} \mathbb{Z}}} \frac{m+n_{1}+k}{N_{1}}
\end{gathered}
$$

Using

$$
\sum_{j=n}^{m} j=\frac{m(m+1)}{2}-\frac{n(n-1)}{2}
$$

the first summand is

$$
m+n_{1}+\frac{1}{m n_{1} n_{2}}\left(\begin{array}{c}
m n_{1} n_{2} \\
2
\end{array}\right)=n_{1}+m+\frac{m n_{1} n_{2}-1}{2} .
$$


For the second summand, we look for $0 \leq k<m n_{1} n_{2}$ such that if $m+n_{1}+k=m s$ for some $s \in \mathbb{Z}$. The minimum of such $s$ is $\left\lceil\frac{m+n_{1}}{m}\right\rceil=2$, while the maximum is

$$
\left\lfloor\frac{m+n_{1}+m n_{1} n_{2}-1}{m}\right\rfloor=n_{1} n_{2}+1
$$

Hence, the second term is

$$
-\sum_{s=2}^{n_{1} n_{2}+1} \frac{s}{n_{1} n_{2}}=-\frac{\left(n_{1} n_{2}+2\right)\left(n_{1} n_{2}+1\right)}{2 n_{1} n_{2}}+\frac{1}{n_{1} n_{2}}=-\frac{n_{1} n_{2}+3}{2} .
$$

For the third term, we proceed in the same way; the extremities are

$$
\left\lceil\frac{m+n_{1}}{n_{1}}\right\rceil=2+\tilde{q}, \quad\left\lfloor\frac{m+n_{1}+m n_{1} n_{2}-1}{n_{1}}\right\rfloor=1+m n_{2}+\tilde{q}
$$

the third term is

$$
-\sum_{s=2+\tilde{q}}^{m n_{2}+\tilde{q}+1} \frac{s}{m n_{2}}=-\frac{\left(m n_{2}+\tilde{q}+2\right)\left(m n_{2}+\tilde{q}+1\right)-(\tilde{q}+2)(\tilde{q}+1)}{2 m n_{2}}=-\frac{m n_{2}+2 \tilde{q}+3}{2} .
$$

For the fourth term the extremities are

$$
\left\lceil\frac{m+n_{1}}{m n_{1}}\right\rceil=1, \quad\left\lfloor\frac{m+n_{1}+m n_{1} n_{2}-1}{m n_{1}}\right\rfloor=n_{2} ;
$$

the fourth term is

$$
\sum_{s=1}^{n_{2}} \frac{s}{n_{2}}=\frac{n_{2}+1}{2}
$$

As a consequence,

$$
\sum_{\beta_{i} \in B_{1}} \beta_{i}=n_{1}+m-\tilde{q}-3+n_{2} \frac{(m-1)\left(n_{1}-1\right)}{2}
$$

For

$$
B_{2}:=\left\{\sigma=\frac{\left(m+n_{1}\right) n_{2}+q+k}{n_{2} \underbrace{\left(m n_{1} n_{2}+q\right)}_{D}} \mid 0 \leq k<n_{2} D \text { and } n_{2} \sigma, D \sigma \notin \mathbb{Z}\right\} .
$$

we decompose $\sum_{\beta_{i} \in B_{2}} \beta_{i}$ again in four terms. For the first one, we have

$$
\left(m+n_{1}\right) n_{2}+q+\frac{n_{2}\left(m n_{1} n_{2}+q\right)-1}{2} .
$$


For the next terms we proceed as in the case of the first exponent. The limits of the second term are:

$$
\begin{gathered}
\left\lceil\frac{\left(m+n_{1}\right) n_{2}+q}{n_{2}}\right\rceil=m+n_{1}+h+1, \\
\left\lfloor\frac{\left(m+n_{1}\right) n_{2}+q+n_{2}\left(m n_{1} n_{2}+q\right)-1}{n_{2}}\right\rfloor=m n_{1} n_{2}+q+m+n_{1}+h ;
\end{gathered}
$$

the second term is

$$
-\sum_{s=m+n_{1}+h+1}^{m n_{1} n_{2}+q+m+n_{1}+h} \frac{s}{m n_{1} n_{2}+q}=-\frac{m n_{1} n_{2}+q+2\left(m+n_{1}+h\right)+1}{2}
$$

The limits of the third term are:

$$
\begin{gathered}
\left\lceil\frac{\left(m+n_{1}\right) n_{2}+q}{m n_{1} n_{2}+q}\right\rceil=1, \\
\left\lfloor\frac{\left(m+n_{1}\right) n_{2}+q+n_{2}\left(m n_{1} n_{2}+q\right)-1}{m n_{1} n_{2}+q}\right\rfloor=n_{2} ;
\end{gathered}
$$

the third term is

$$
-\sum_{s=1}^{n_{2}} \frac{s}{n_{2}}=-\frac{n_{2}+1}{2}
$$

Finally, the limits for the fourth term are

$$
\begin{gathered}
\left\lceil\frac{\left(m+n_{1}\right) n_{2}+q}{n_{2}\left(m n_{1} n_{2}+q\right)}\right\rceil=1, \\
\left\lfloor\frac{\left(m+n_{1}\right) n_{2}+q+n_{2}\left(m n_{1} n_{2}+q\right)-1}{n_{2}\left(m n_{1} n_{2}+q\right)}\right\rfloor=1 ;
\end{gathered}
$$

the fourth term is 1 . Then,

$$
\sum_{\beta_{i} \in B_{2}} \beta_{i}=\left(m+n_{1}\right) n_{2}+q-\left(m+n_{1}+h\right)-1+\frac{\left(n_{2}-1\right)\left(m n_{1} n_{2}+q-1\right)}{2} .
$$

Recall that

$$
\mu=n_{2}\left(n_{1}-1\right)(m-1)+\left(n_{2}-1\right)\left(m n_{1} n_{2}+q-1\right) .
$$

The sum of the exponents is

$$
\frac{\mu}{2}+\left(m+n_{1}\right) n_{2}+q-\tilde{q}-h-4
$$

while the sum of the spectral numbers is $\mu$. Then, its difference is

$$
\frac{\mu}{2}-\left(m+n_{1}\right) n_{2}-q+\tilde{q}+h+4
$$

as stated. 
Proposition 3.2. Let $f$ be an irreducible germ of plane curve whose characteristic sequence is $C S_{n_{1}, m}^{n_{2}, q}$ satisfying (15). Then the following lower bound for $\operatorname{dim} \tilde{H}_{0}^{\prime \prime} / H_{0}^{\prime \prime}$ is obtained:

$$
\left(n_{2}-1\right)(m-1)\left(n_{1}-1\right) \leq \operatorname{dim} \tilde{H}_{0}^{\prime \prime} / H_{0}^{\prime \prime}
$$

Proof. We are going to count some spectral numbers $\alpha \in A_{1}^{\perp} \cup A_{2}^{\perp}$ such that $\alpha-1 \in B_{11} \cup B_{21}$. This number is a lower bound for $\operatorname{dim} \tilde{H}_{0}^{\prime \prime} / H_{0}^{\prime \prime}$.

Let $\alpha \in A_{1}^{\perp}$; we have

$$
\alpha-1=1-\frac{1}{n_{2}}\left(\frac{i}{n_{1}}+\frac{j}{m}+r\right)
$$

with $\frac{i}{n_{1}}+\frac{j}{m}<1, r<n_{2}$. Assume that $r<n_{2}-1$ :

$$
\alpha-1=\frac{n_{1} m\left(n_{2}-r-1\right)+n_{1} m-m i-n_{1} j}{n_{1} n_{2} m} \in B_{11}
$$

since the numerator is in $\Gamma$. Hence, we have found at least $\left(n_{2}-1\right) \frac{(m-1)\left(n_{1}-1\right)}{2}$ such numbers.

Let $\alpha \in A_{2}^{\perp}$ :

$$
\alpha-1=1-\left(\frac{i}{n_{2}}+\frac{j}{n_{1} n_{2} m+q}\right)=\frac{\left(n_{2}-i-1\right)\left(n_{1} n_{2} m+q\right)+q+n_{2}\left(n_{1} m-j\right)}{n_{2}\left(n_{1} n_{2} m+q\right)} .
$$

A necessary (and by the way sufficient condition) for $\alpha-1 \in B_{21}$ is the existence of $\beta_{1}, \beta_{2} \in \mathbb{Z}_{\geq 1}$ such that $m n_{1}-j=m \beta_{1}+n_{1} \beta_{2}$. We found another set of $\left(n_{2}-1\right) \frac{(m-1)\left(n_{1}-1\right)}{2}$ such numbers.

Remark 3.3. In [11, Proposition 3.5], another bound for $\operatorname{dim} \tilde{H}_{0}^{\prime \prime} / H_{0}^{\prime \prime}$ is given which depends on the analytical properties of the germ, namely, $\mu-\tau \leq \operatorname{dim} \tilde{H}_{0}^{\prime \prime} / H_{0}^{\prime \prime}$ which yields the following bound for the Tjurina number:

$$
\tau \geq \frac{\mu}{2}+n_{2}\left(m+n_{1}\right)+q-\tilde{q}-h-4
$$

\section{EXAMPLES}

Example 4.1. We consider the case studied by Tamaki Yano, that is the characteristic sequence $(4,6,6+q)$, i.e. $n_{1}=2, m=3, n_{2}=2$ and $q=q$. T. Yano in 1983 claimed the proof of his conjecture in this case, but referred to a non 
published article. The set of spectral numbers is

$$
\begin{gathered}
\operatorname{Spec}(f)=\overbrace{\left\{\frac{5}{12}, \frac{11}{12}\right\}}^{A_{1}} \cup \overbrace{\left\{\frac{19}{12}, \frac{13}{12}\right\}}^{A_{1}^{\perp}} \cup \\
\{\frac{12+q+2 j}{\left\{\frac{A_{2}}{2(12+q)} \mid 0<j \leq 6+\left\lfloor\frac{q}{2}\right\rfloor\right\}} \cup \overbrace{\left\{\frac{3(12+q)-2 j}{2(12+q)} \mid 0<j \leq 6+\left\lfloor\frac{q}{2}\right\rfloor\right\}}^{A_{2}^{\perp}} ;
\end{gathered}
$$

it is not hard to see that

$$
A_{2} \cup A_{2}^{\perp}=\left\{\frac{14+q}{2(12+q)}, \frac{16+q}{2(12+q)}, \ldots, \frac{34+3 q}{2(12+q)}\right\}
$$

is a gap-free arithmetic sequence with step $\frac{1}{12+q}$. The set of spectral numbers $\alpha$ such that $\alpha-1 \geq 5 / 12$ is

$$
\left.\operatorname{Spec}(f) \backslash \mathcal{E}=\left\{\frac{19}{12}\right\} \cup\left\{\frac{12+q+2 j}{2(12+q)}\right\rceil\left\lceil\frac{11(12+q)}{12}\right\rceil \leq j<12+q\right\} .
$$

Recall that we cannot ensure for these spectral numbers to be exponents. We also have

$$
B_{1}=B_{11}=\left\{\frac{5}{12}, \frac{7}{12}, \frac{11}{12}, \frac{13}{12}\right\}, \quad B_{2}=B_{21}=\left\{\frac{10+q+2 k}{2(12+q)} \mid 0 \leq k \leq 11+q, k \neq 1\right\} .
$$

Note also that $\frac{7}{12}$ and $\frac{10+q}{2(12+q)}$ are the only $b$-exponents which are not spectral numbers.

As a consequence, we derive the following result.

Theorem 4.2. For any $f$ with characteristic sequence $(4,6,6+q), B_{1} \cup B_{2}$ is its set of b-exponents (and also the set of roots of the Bernstein polynomial). Moreover, $\operatorname{dim} \tilde{H}_{0}^{\prime \prime} / H_{0}^{\prime \prime}=2$.

Note that the bounds of Propositions 3.1 and 3.2 are equal for any $f$. From Hertling-Stahlke bound of Remark 3.3 we get that $\tau \geq \mu-2$. The value of $\tau$ equals $12+2 q$ and it is constant in the whole stratum, see [17].

Example 4.3. We consider the case of characteristic sequence $(6,9,9+q)$ with $q=1+3 k, k \in \mathbb{N}$. In this case $n_{1}=2, m=3$ and $n_{2}=3$. We have

$$
\begin{gathered}
A_{1}=\left\{\frac{5}{18}, \frac{11}{18}, \frac{17}{18}\right\}, \quad A_{1}^{\perp}=\left\{\frac{31}{18}, \frac{25}{18}, \frac{19}{18}\right\}, \\
A_{2}=\left\{\frac{19+3 k+3 j}{3(19+3 k)} \mid 0<j \leq 12+2 k\right\} \cup\left\{\frac{2(19+3 k)+3 j}{3(19+3 k)} \mid 0<j \leq 6+k\right\}, \\
A_{2}^{\perp}=\left\{\frac{5(19+3 k)-3 j}{3(19+3 k)} \mid 0<j \leq 12+2 k\right\} \cup\left\{\frac{4(19+3 k)-3 j}{3(19+3 k)} \mid 0<j \leq 6+k\right\} .
\end{gathered}
$$


In fact

$$
A_{2} \cup A_{2}^{\perp}=\left\{\frac{22+3 k}{3(19+3 k)}, \ldots, \frac{73+12 k}{3(19+3 k)}\right\} \cup\left\{\frac{41+6 k}{3(19+3 k)}, \ldots, \frac{92+15 k}{3(19+3 k)}\right\}
$$

is the union of two step- $\frac{1}{19+3 k}$ arithmetic sequences. The set $\mathcal{E}$ is determined by

$$
\begin{aligned}
\operatorname{Spec}(f) \backslash \mathcal{E}= & \left\{\frac{25}{18}, \frac{31}{18}\right\} \cup\left\{\frac{2(19+3 k)+3 j}{3(19+3 k)} \mid\left\lceil\frac{11(19+3 k)}{18}\right\rceil \leq j<19+3 k\right\} \\
& \cup\left\{\frac{19+3 k+3 j}{3(19+3 k)} \mid\left\lceil\frac{17(19+3 k)}{18}\right\rceil \leq j<19+3 k\right\} .
\end{aligned}
$$

The sets of generic $b$-exponents are

$$
\begin{aligned}
& B_{1}=B_{11}=\left\{\frac{5}{18}, \frac{7}{18}, \frac{11}{18}, \frac{13}{18}, \frac{17}{18}, \frac{19}{18}\right\} \\
& B_{2}=\left\{\frac{16+3 k+3 j}{3(19+3 k)} \mid 0 \leq j \underset{j \neq 1}{<19+3 k}\right\} \cup\left\{\frac{17+3 k+3 j}{3(19+3 k)} \mid 0 \leq j \underset{j \neq k+7}{<19+3 k}\right\} \text {, } \\
& B_{22}=\left\{\frac{17+3 k+3 j}{3(19+3 k)} \mid 0 \leq j<k+6\right\} \text {. }
\end{aligned}
$$

Note that $\frac{7}{18}, \frac{13}{18}$ are the generic $b$-exponents in $B_{1}$ which are not spectral numbers. For $B_{2}$ this is the case for

$$
\left\{\frac{16+3 k}{3(19+3 k)}\right\} \cup\left\{\frac{17+3 k+3 j}{3(19+3 k)} \mid 0 \leq j<k+7\right\} .
$$

In particular, generically $\operatorname{dim} \tilde{H}_{0}^{\prime \prime} / H_{0}^{\prime \prime}=10+k$. Among them, note that $\frac{7}{18}, \frac{13}{18} \in$ $B_{11}$ and

$$
\frac{16+3 k}{3(19+3 k)}, \frac{35+6 k}{3(19+3 k)} \in B_{21}
$$

moreover they are the only common roots which are not spectral numbers, hence $\operatorname{dim} \tilde{H}_{0}^{\prime \prime} / H_{0}^{\prime \prime} \geq 4$. We do not know if the equality can be reached.

The elements of $\operatorname{Spec}(f) \backslash \mathcal{E}$ that can jump to give generic roots of the Bernstein polynomial are

$$
\left\{\frac{25}{18}, \frac{31}{18}\right\} \cup\left\{\frac{2(19+3 k)+3 j}{3(19+3 k)} \mid\left\lceil\frac{11(19+3 k)}{18}\right\rceil \leq j<19+3 k\right\} \cup\left\{\frac{73+12 k}{3(19+3 k)}\right\} .
$$

Consider

$$
f_{t}:=\left(x^{2}-y^{3}\right)^{3}+x^{5+k} y^{2}+t\left(x^{2}-y^{3}\right) x^{5} y^{k-1}
$$

where $t$ is chosen such that such that $f_{t}$ is of type $(6,9,10+3 k)^{-}$. Let $\beta=$ $\frac{3\left(3 \beta_{1}+2 \beta_{2}\right)+3 k+2}{3(19+3 k)} \in B_{2}$ and in (4.1), i.e.

$$
\frac{3\left(3 \beta_{1}+2 \beta_{2}\right)+3 k+2}{3(19+3 k)}=\frac{17+3 k+3 j}{3(19+3 k)} \Leftrightarrow 3 \beta_{1}+2 \beta_{2}=5+j .
$$


Since we need $\beta_{1}, \beta_{2} \geq 1$, all the cases are included but $j=1$. We are going to prove $-\beta$ is a root of $f_{t}$ if $t \neq 0$, and as a consequence, for $t \neq 0$ we have $\operatorname{dim} \tilde{H}_{0}^{\prime \prime} / H_{0}^{\prime \prime} \geq 9+k$.

We consider the polynomials $\tilde{f}, \tilde{\tilde{f}}, \hat{f}, \hat{\hat{f}}, f_{1}, f_{2}$ :

$$
\begin{gathered}
\tilde{f}(x, y)=\left(x^{6}-y^{6}\right)^{3}+x^{15+3 k} y^{4}+t\left(x^{6}-y^{6}\right) x^{15} y^{2 k-2}, \\
\tilde{\tilde{f}}(x, y)=\left(1-y^{6}\right)^{3}+x^{1+3 k} y^{4}+t\left(1-y^{6}\right) x^{1+2 k} y^{2 k-2}, \\
\hat{f}(x, y)=\left(1-(1-y)^{6}\right)^{3}+x^{1+3 k}(1-y)^{4}+t\left(1-(1-y)^{6}\right) x^{1+2 k}(1-y)^{2 k-2}= \\
y^{3}\left(6^{3}+\ldots\right)+x^{1+3 k}(1-\ldots)+t x^{1+2 k} y(6+\ldots), \\
\hat{\hat{f}}(x, y)=y^{3(1+3 k)}\left(6^{3}+\ldots\right)+x^{3(1+3 k)}(1-\ldots)+t x^{3(1+2 k)} y^{1+3 k}(6+\ldots), \\
f_{1}(x, y)=y^{3(1+3 k)}\left(6^{3}+\ldots\right)+(1-\ldots)+t x y^{1+3 k}(6+\ldots), \\
f_{2}(x, y)=\left(6^{3}+\ldots\right)+x^{3(1+3 k)}(1-\ldots)+t x^{3(1+2 k)} y(6+\ldots) .
\end{gathered}
$$

We have

$\operatorname{Res}_{s=-\beta} I_{-}\left(f, \beta_{1}, \beta_{2}, 0\right)(s)=\frac{1}{3(1+3 k)}\left(G_{h_{1,-\beta, x}^{1}}(1+3 k)+G_{h_{1,-\beta, y}^{2}}\left(2\left(-18 \beta+3 \beta_{1}+2 \beta_{2}\right)\right)\right.$,

where

$$
\begin{aligned}
& h_{1,-\beta, x}^{1}(y)=\frac{\partial f_{1}^{-\beta}}{\partial x}(0, y)=-6 \beta t y^{1+3 k}\left(6^{3} y^{3(1+3 k)}+1\right)^{-\beta-1} \\
& h_{1,-\beta, y}^{2}(x)=\frac{\partial f_{2}^{-\beta}}{\partial y}(x, 0)=-6 \beta t x^{3(1+2 k)}\left(6^{3}+x^{(1+3 k) 3}\right)^{-\beta-1}
\end{aligned}
$$

If $t$ is algebraic (and $t \neq 0$ ), the above residue is transcendental. Hence, we deduce that these values are roots of the Bernstein polynomial for these values of $t$. Moreover, since the Bernstein-polynomial stratification is algebraic, we deduce that this is the case for $t \neq 0$. Note that in this case, for $k=0$, and for random values of $t$, the Tjurina number equals 40 , while for $t=0$, the value is 41 . Hence the Tjurina number is not constant in the $\mu$-constant stratum. 
Example 4.4. Consider the characteristic sequence $(8,10,10+q)$, where $(q, 2)=$ $1,(q, 5)=1$. In this case $n_{1}=4, m=5$ and $n_{2}=2$. We have

$$
\begin{gathered}
A_{1}=\left\{\frac{9}{40}, \frac{13}{40}, \frac{14}{40}, \frac{17}{40}, \frac{18}{40}, \frac{19}{40}, \frac{29}{40}, \frac{33}{40}, \frac{34}{40}, \frac{37}{40}, \frac{38}{40}, \frac{39}{40}\right\} \\
A_{1}^{\perp}=\left\{\frac{71}{40}, \frac{67}{40}, \frac{66}{40}, \frac{63}{40}, \frac{62}{40}, \frac{61}{40}, \frac{51}{40}, \frac{47}{40}, \frac{46}{40}, \frac{43}{40}, \frac{42}{40}, \frac{41}{40}\right\} \\
A_{2}=\left\{\frac{40+q+2 j}{2(40+q)} \mid 0<j \leq 20+\left\lfloor\frac{q}{2}\right\rfloor\right\} \\
A_{2}^{\perp}=\left\{\frac{3(40+q)-2 j}{2(40+q)} \mid 0<j \leq 20+\left\lfloor\frac{q}{2}\right\rfloor\right\}
\end{gathered}
$$

Note that

$$
A_{2} \cup A_{2}^{\perp}=\left\{\frac{42+q}{2(40+q)}, \frac{44+q}{2(40+q)}, \ldots, \frac{118+3 q}{2(40+q)}\right\}
$$

is a step- $\frac{1}{40+q}$ arithmetic sequence.

Then $\operatorname{Spec}(f) \backslash \mathcal{E}$ is

$$
\left\{\frac{51}{40}, \frac{61}{40}, \frac{62}{40}, \frac{63}{40}, \frac{66}{40}, \frac{67}{40}, \frac{71}{40}\right\} \cup\left\{\frac{40+q+2 j}{2(40+q)} \mid 29+\left\lceil\frac{29}{40} q\right\rceil \leq j \leq 20+\left\lfloor\frac{q}{2}\right\rfloor\right\} .
$$

With this data

$$
B_{1}=\left\{\frac{9+4 \ell}{40} \mid 0 \leq \underset{\ell \neq 4}{\ell \leq 8}\right\} \cup\left\{\frac{10+4 \ell}{40} \mid 1 \leq \underset{\ell \neq 5}{\ell \leq 9}\right\} \cup\left\{\frac{11+4 \ell}{40} \mid 0 \leq \underset{\ell \neq 1,6}{\leq \leq 9}\right\},
$$

where $B_{12}=\left\{\frac{11}{40}\right\}$, and

$$
\begin{gathered}
B_{2}=\left\{\frac{18+q+2 \ell}{2(40+q)} \mid 0 \leq \ell<40+q, \ell \neq 11\right\}, \\
B_{22}=\left\{\frac{20+q}{2(40+q)}, \frac{22+q}{2(40+q)}, \frac{24+q}{2(40+q)}, \frac{30+q}{2(40+q)}, \frac{32+q}{2(40+q)}\right\} .
\end{gathered}
$$

To get the Bernstein polynomial for any function with characteristic sequence $(8,10,10+q)$, we only have to check for the 6 elements of $B_{12} \cup B_{22}$ if they are roots (recall that the Milnor number is $63+q$ ). Let us study the generic b-exponents which are not spectral numbers:

$$
\left\{\frac{21}{40}, \frac{22}{40}, \frac{26}{40}, \frac{11}{40}, \frac{23}{40}, \frac{27}{40}, \frac{31}{40}\right\} \cup\left\{\frac{18+q+2 \ell}{2(40+q)} \mid 0 \leq \ell \leq 10\right\} \supset B_{12} \cup B_{22} .
$$

We get $12 \leq \operatorname{dim} \tilde{H}_{0}^{\prime \prime} / H_{0}^{\prime \prime} \leq 18$. We shall show, for $q=7$, that there exists an $f$ with characteristic sequence $(8,10,10+q)$ such that $12=\operatorname{dim} \tilde{H}_{0}^{\prime \prime} / H_{0}^{\prime \prime}$. Consider

$$
f_{ \pm}(x, y)=\left(x^{4} \pm y^{5}\right)^{2}+x^{7} y^{3}+t x^{6} y^{6} \text {. }
$$


We assume that $f_{ \pm}$has type $(8,10,10+q)^{ \pm}$. Let

$$
\begin{gathered}
\tilde{f}_{+}(x, y)=f_{+}\left(x^{4}, y^{5}\right)=\left(x^{20}+y^{20}\right)^{2}+x^{35} y^{12}+t x^{30} y^{24} \\
f_{+1}(x, y)=\left(1+y^{20}\right)^{2}+x^{7} y^{12}+t x^{14} y^{24} \\
f_{+2}(x, y)=\left(x^{20}+1\right)^{2}+x^{35} y^{7}+t x^{30} y^{14}
\end{gathered}
$$

Let $\beta=\frac{11}{40}=\frac{(5+4)+2}{40}$. The residue is

$$
\operatorname{Res}_{s=-\beta} I\left(f_{+}, 1,1\right)(s)=\frac{1}{80}\left(G_{h_{2,-\beta, x}^{1}}(4)+G_{h_{2,-\beta, y}^{2}}(5)\right)
$$

where

$$
h_{2,-\beta, x}^{1}(y)=\frac{\partial^{2} f_{1}^{-\beta}}{\partial x^{2}}(0, y)=0, \quad h_{2,-\beta, y}^{2}(x)=\frac{\partial^{2} f_{2}^{-\beta}}{\partial y^{2}}(x, 0)=0 .
$$

Then $\operatorname{Res}_{s=-\beta} I\left(f_{+}, 1,1\right)(s)=0$; moreover, with the same ideas as in the proof of Claim 2.8 we have that $\forall\left(\beta_{1}, \beta_{2}\right)$, $\operatorname{Res}_{s=-\beta} I\left(f_{+}, \beta_{1}, \beta_{2}\right)(s)=0$.

Consider now $\beta=\frac{51}{40}=\frac{5 \cdot 3+4 \cdot 9}{40}$. We know that $I\left(f_{+}, 3,9\right)(s)$ has a pole for $s=-\beta$ with transcendental residue. Combining the two facts, by Theorem 1.13 , $-\frac{51}{40}$ is a zero of the Bernstein polynomial of $f_{+}$and $-\frac{11}{40}$ is not. Since $f_{+}(x, y)=$ $f_{-}(-x,-y)$, we deduce the same property for $f_{-}$.

It remains to study the cases in $B_{22}$, i.e., the set $\left\{\frac{27}{94}, \frac{29}{94}, \frac{31}{94}, \frac{37}{94}, \frac{39}{94}\right\}$, with $k=$ $2,4,6,12,14$. Since the generators of $\Gamma$ are $8,10,47$, only the following suitable combinations of $\beta_{1}, \beta_{2}, \beta_{3}, \nu$ (see proof of Claim 2.10) are available:

\begin{tabular}{|c|c|c|c|c|c|c|}
\hline$k$ & $\beta_{1}$ & $\beta_{2}$ & $\beta_{3}$ & $\nu$ & $i$ & $\nu_{i}$ \\
\hline 2 & 1 & 1 & 0 & 2 & 1 & 2 \\
\hline 4 & 1 & 1 & 0 & 4 & 1 & 4 \\
\hline 6 & 1 & 1 & 0 & 6 & 1 & 6 \\
\hline 12 & 1 & 1 & 0 & 12 & 1 & 12 \\
\hline 12 & 1 & 1 & 0 & 12 & 2 & 5 \\
\hline 12 & 2 & 1 & 0 & 4 & 1 & 4 \\
\hline 12 & 1 & 2 & 0 & 2 & 1 & 2 \\
\hline 14 & 1 & 1 & 0 & 14 & 1 & 14 \\
\hline 14 & 1 & 1 & 0 & 14 & 2 & 7 \\
\hline 14 & 1 & 1 & 0 & 14 & 3 & 0 \\
\hline 14 & 2 & 1 & 0 & 6 & 1 & 6 \\
\hline 14 & 1 & 2 & 0 & 4 & 1 & 4 \\
\hline
\end{tabular}


Let us compute the polynomials appearing in the different steps of the process:

$$
\begin{gathered}
f(x, y)=\left(x^{4}-y^{5}\right)^{2}+x^{7} y^{3}+t x^{6} y^{6} \\
\tilde{f}(x, y)=\left(x^{20}-y^{20}\right)^{2}+x^{35} y^{12}+t x^{30} y^{24} \\
\tilde{\tilde{f}}(x, y)=\left(1-y^{20}\right)^{2}+x^{7} y^{12}+t x^{14} y^{24} \\
\hat{f}(x, y)=y^{2} H_{20}(y)^{2}+x^{7}(1-y)^{12}+t x^{14}(1-y)^{24}, \\
\hat{\hat{f}}(x, y)=y^{14} H_{20}\left(y^{7}\right)^{2}+x^{14}\left(1-y^{7}\right)^{12}+t x^{28}\left(1-y^{7}\right)^{24}, \\
f_{1}(x, y)=y^{14} H_{20}\left(x^{7} y^{7}\right)^{2}+\left(1-x^{7} y^{7}\right)^{12}+t x^{14}\left(1-x^{7} y^{7}\right)^{24}, \\
f_{2}(x, y)=H_{20}\left(y^{7}\right)+x^{14}\left(1-y^{7}\right)^{12}+t x^{28} y^{14}(1-y)^{24} .
\end{gathered}
$$

where $y H_{n}(y)=\left(1-(1-y)^{n}\right), H_{n}(0)=n$. From these data it is easy to check that

$$
\frac{\partial^{N} f_{1}}{\partial x^{N}}(0, y)= \begin{cases}20^{2} y^{14}+1, & \text { if } N=0 \\ -20160 y^{7}\left(1900 y^{14}+3\right), & \text { if } N=7 \\ 87178291200\left(81700 y^{28}+66 y^{14}+t\right), & \text { if } N=14 \\ 0 & \text { if } \frac{N}{7} \notin \mathbb{Z} .\end{cases}
$$

and

$$
\frac{\partial^{N} f_{2}}{\partial y^{N}}(x, 0)= \begin{cases}20^{2}+x^{14}, & \text { if } N=0 \\ -20160\left(1900+3 x^{14}\right), & \text { if } N=7 \\ 87178291200\left(81700+66 x^{14}+t x^{28}\right), & \text { if } N=14 \\ 0 & \text { if } \frac{N}{7} \notin \mathbb{Z} .\end{cases}
$$

With the same ideas

$$
\frac{\partial^{N} f_{1}^{-\beta}}{\partial x^{N}}(0, y)= \begin{cases}\left(20^{2} y^{14}+1\right)^{-\beta}, & \text { if } N=0 \\ 20160 \beta y^{7}\left(1900 y^{14}+3\right)\left(20^{2} y^{14}+1\right)^{-\beta-1}, & \text { if } N=7 \\ -87178291200 \beta\left(81700 y^{28}+66 y^{14}+t\right)\left(20^{2} y^{14}+1\right)^{-\beta-1}+ & \\ 697426329600 \beta(\beta+1) y^{14}\left(1900 y^{14}+3\right)^{2}\left(20^{2} y^{14}+1\right)^{-\beta-2} & \text { if } N=14 \\ 0 & \text { if } \frac{N}{7} \notin \mathbb{Z} .\end{cases}
$$


and

$$
\frac{\partial^{N} f_{2}^{-\beta}}{\partial y^{N}}(x, 0)= \begin{cases}\left(20^{2}+x^{14}\right)^{-\beta}, & \text { if } N=0 \\ 20160 \beta\left(1900+3 x^{14}\right)\left(20^{2}+x^{14}\right)^{-\beta-1}, & \text { if } N=7 \\ -87178291200 \beta\left(81700+66 x^{14}+t x^{28}\right)\left(20^{2}+x^{14}\right)^{-\beta-1}+ & \\ 697426329600 \beta(\beta+1)\left(1900+3 x^{14}\right)^{2}\left(20^{2}+x^{14}\right)^{-\beta-2} & \text { if } N=14 \\ 0 & \text { if } \frac{N}{7} \notin \mathbb{Z} .\end{cases}
$$

It becomes obvious that all the residues vanish for $k=2,4,6,12$. The residue also vanishes for $k=14$ and $\left(\beta_{1}, \beta_{2}\right)=(2,1),(1,2)$. Let us study the case $k=14$, i.e., $\beta=\frac{39}{94}$, with $\left(\beta_{1}, \beta_{2}\right)=(1,1)$. Note that for $\beta_{2}=1$,

$$
h_{4}(y)=(1-y)^{3}=1-3 y+3 y^{2}-y^{3} .
$$

Hence,

$$
\begin{gathered}
14 \operatorname{Res}_{s=-\beta} I_{-}(f, 1,1,0)(s)=\frac{1}{14 !}\left(G_{h_{14,-\beta, x}^{1}}(7)+G_{h_{14,-\beta, y}^{2}}\left(-\frac{714}{47}\right)\right) \\
-\frac{3}{7 !}\left(G_{h_{7,-\beta, x}^{1}}(14)+G_{h_{7,-\beta, y}^{2}}\left(-\frac{714}{47}\right)\right)+3\left(G_{h_{0,-\beta, x}^{1}}(21)+G_{h_{0,-\beta, y}^{2}}\left(-\frac{714}{47}\right)\right)
\end{gathered}
$$

We find the values of $h_{1}(k,-\beta, x)$ and $h_{2}(k,-\beta, y)$ in (4.2) and (4.3).

We can prove that the pole at $s=-\beta$ of $I_{-}(f, 1,1)(s)$ is a polynomial of degree 1 in $t$ and hence there is a value of $t$ for which the residue vanishes. Moreover

$$
\operatorname{Res}_{s=-\beta} I_{-}(f, 1,1,0)(s)=\frac{(136 t-63)}{447440} \boldsymbol{B}\left(-\frac{4}{47}, \frac{1}{2}\right) .
$$

In particular, none of the above elements are roots of the Bernstein polynomial of $f_{-}$for $t=\frac{63}{136}$. For $t \neq \frac{63}{136},-\frac{39}{94}$ is such a root but not for $t=\frac{63}{136}$. This can be confirmed using checkRoot of [14] in Singular [9], inside [26]. Moreover, it can be proved that for general $t$ (including $\frac{63}{136}$ ) the Tjurina number equals the expected value for Hertling-Stahlke bound, i.e., 58; using [17] the values of Tjurina number are constant in these $\mu$-constant strata, namely they equal $51+q$. In particular, Bernstein and Tjurina stratifications do not coincide.

\section{Appendix A. TeChnical proofs}

Proof of Claim 2.8. Let us recall that $\beta=\frac{m+n_{1}+k}{m n_{1} n_{2}}$ and $k \notin \Gamma_{1}$.

Let $\left(\beta_{1}, \beta_{2}\right) \in \mathbb{Z}_{\geq 1}^{2}$. If $\beta_{1} m+\beta_{2} n_{1}>m+n_{1}+k$, the greatest pole of $I\left(f, \beta_{1}, \beta_{2}\right)(s)$ is smaller than $-\beta$ and the statement holds trivially for any $f$. 
We want to fix our attention on the couples $\left(\beta_{1}, \beta_{2}\right) \in \mathbb{Z}_{\geq 1}^{2}$ such that $\beta_{1} m+$ $\beta_{2} n_{1} \leq m+n_{1}+k$. There is a finite number of such couples which will be characterized in the following paragraphs.

Since $k \notin \Gamma_{1}$, and from its properties, we know that $k \leq m n_{1}-m-n_{1}$. We write

$$
k=m i_{0}+n_{1} j_{0}-m n_{1}, \quad 1 \leq i_{0}<n_{1}, \quad 1 \leq j_{0}<m
$$

Moreover the pair of positive integers $\left(i_{0}, j_{0}\right)$ is unique. Let us assume the existence of another solution $\left(i_{1}, j_{1}\right)$, such that $i_{1}>i_{0}$; then $i_{1}=i_{0}+n_{1} v, v \in \mathbb{Z}_{>0}$, i.e., $i_{1}>n_{1}$, leading to a contradiction.

We are going to prove also that $\beta_{1} \leq i_{0}$ and $\beta_{2} \leq j_{0}$. Let us assume that $\beta_{1}>i_{0}$. Then

$$
\begin{gathered}
\left(i_{0}+1\right) m+\beta_{2} n_{1}<\beta_{1} m+\beta_{2} n_{1} \leq m+n_{1}+k=m\left(i_{0}+1\right)+n_{1}\left(j_{0}+1\right)-m n_{1} \\
\Longrightarrow \beta_{2}+m \leq j_{0}+1<m+1
\end{gathered}
$$

which is a contradiction. We are going to enumerate these couples $\left(\beta_{1}, \beta_{2}\right)$.

Let us define $\ell_{i j}:=m i+n_{1} j-n_{1} m$ and consider

$$
\left\{\ell_{i j} \mid \ell_{i j} \geq 1,1 \leq i \leq i_{0}, 1 \leq j \leq j_{0}\right\}=\left\{\ell_{1}, \ldots, \ell_{r}\right\}, \quad \ell_{1}<\cdots<\ell_{r}=k .
$$

Let

$$
\ell_{p}=m i_{p}+n_{1} j_{p}-m n_{1}, \quad i_{p}, j_{p} \text { well-defined, for } 1 \leq p \leq r .
$$

For each $p$ we can write

$$
\beta=\frac{m \overbrace{\left(1+i_{0}-i_{p}\right)}^{\beta_{1 p}}+n_{1} \overbrace{\left(1+j_{0}-j_{p}\right)}^{\beta_{2 p}}+\ell_{p}}{n_{1} n_{2} m} ;
$$

note that $\beta_{1 r}=\beta_{2 r}=1$ and $1 \leq \beta_{1 p} \leq i_{0}, 1 \leq \beta_{2 p} \leq j_{0}$. It is easy to prove that $\left\{\left(\beta_{1 p}, \beta_{2 p}\right) \mid 1 \leq p \leq r\right\}=\left\{\left(\beta_{1}, \beta_{2}\right) \in \mathbb{Z}_{\geq 1} \times \mathbb{Z}_{\geq 1} \mid \beta_{1} m+\beta_{2} n_{1} \leq m+n_{1}+k\right\}$. These $r$ pairs are exactly the ones for which we need to prove the statement.

Define

$$
f_{\mathbf{t}}(x, y):=\left(x^{n_{1}}+y^{m}+\sum_{p=1}^{r} t_{p} x^{i_{p}} y^{j_{p}}\right)^{n_{2}}+x^{a} y^{b} .
$$

with $m a+n_{1} b=q+m n_{1} n_{2}$ and $\mathbf{t}=\left(t_{1}, \ldots, t_{r}\right) \in \mathbb{R}^{r}$ such that $f_{\mathbf{t}}$ is of type $\left(n_{1} n_{2}, m n_{2}, m n_{2}+q\right)^{+}$. By Proposition 1.8 one has

$$
\tilde{f}_{\mathbf{t}}(x, y)=\left(x^{m n_{1}}+y^{m n_{1}}+\sum_{p=1}^{r} t_{p} x^{m i_{p}} y^{n_{1} j_{p}}\right)^{n_{2}}+x^{m a} y^{n_{1} b}
$$




$$
\begin{gathered}
f_{\mathbf{t}, 1}(x, y)=\left(1+y^{m n_{1}}+\sum_{p=1}^{r} t_{p} x^{\ell_{p}} y^{n_{1} j_{p}}\right)^{n_{2}}+x^{q} y^{n_{1} b} . \\
f_{\mathbf{t}, 2}(x, y)=\left(x^{m n_{1}}+1+\sum_{p=1}^{r} t_{p} x^{m i_{p}} y^{\ell_{p}}\right)^{n_{2}}+x^{m a} y^{q} .
\end{gathered}
$$

Let us fix $p \in\{1, \ldots, r\}$. To compute the residue of $I_{+}\left(f_{\mathbf{t}}, \beta_{1 p}, \beta_{2 p}\right)(s)$ at $s=-\beta$ we apply equation (1.6) and we get

$$
\rho_{p}:=\operatorname{Res}_{s=-\beta} I_{+}\left(f_{\mathbf{t}}, \beta_{1 p}, \beta_{2 p}\right)(s)=\frac{1}{\ell_{p} ! m n_{1} n_{2}}\left(G_{h_{\ell_{p},-\beta, x}^{1}}\left(n_{1} \beta_{2 p}\right)+G_{h_{\ell_{p},-\beta, y}^{2}}\left(m \beta_{1 p}\right)\right)
$$

where

$$
h_{\ell_{p},-\beta, x}^{1}(y)=\frac{\partial^{\ell_{p}} f_{\mathbf{t}, 1}^{-\beta}}{\partial x^{\ell_{p}}}(0, y) \text { and } h_{\ell_{p},-\beta, y}^{2}(x)=\frac{\partial^{\ell_{p}} f_{\mathbf{t}, 2}^{-\beta}}{\partial y^{\ell_{p}}}(x, 0),
$$

recall that $G_{f}(s)$ is meromorphic continuation of $\int_{0}^{1} f(t) t^{s} \frac{d t}{t}$.

We have

$$
\frac{\partial^{\ell_{p}} f_{\mathbf{t}, 1}^{-\beta}}{\partial x^{\ell_{p}}}(0, y)=\sum_{V=\left(u_{w}\right)_{w=1}^{|V|} \in \mathcal{P}\left(\ell_{p}\right)} D_{V}\left(\prod_{w=1}^{|V|} \frac{\partial^{u_{w}} f_{\mathbf{t}, 1}}{\partial x^{u_{w}}}(0, y)\right)\left(1+y^{m n_{1}}\right)^{-n_{2}(\beta+|V|)}
$$

where

$$
\mathcal{P}\left(\ell_{p}\right)=\left\{V=\left(u_{w}\right)_{w=1}^{|V|} \mid \sum_{w=1}^{|V|} u_{w}=\ell_{p}, u_{1} \leq \cdots \leq u_{|V|}\right\}
$$

and $D_{V} \in \mathbb{Q}$. In the same way,

$$
\frac{\partial^{\ell_{p}} f_{\mathbf{t}, 2}^{-\beta}}{\partial y^{\ell_{p}}}(x, 0)=\sum_{V=\left(u_{w}\right)_{w=1}^{|V|} \in \mathcal{P}\left(\ell_{p}\right)} D_{V}\left(\prod_{w=1}^{|V|} \frac{\partial^{u_{w}} f_{\mathbf{t}, 2}}{\partial y^{u_{w}}}(x, 0)\right)\left(1+x^{m n_{1}}\right)^{-n_{2}(\beta+|V|)} .
$$

Let us study now the $u^{\text {th }} x$-derivative of $f_{\mathbf{t}, 1}$ evaluated at $(0, y)$, i.e., we need to look for the monomials of the type $x^{u} y^{j}$, for any $j$. Hence,

$$
\frac{\partial^{u} f_{\mathbf{t}, 1}}{\partial x^{u}}(0, y)=\delta_{q}^{u} q ! y^{n_{1} b}+\sum_{K=\left(k_{h}\right)_{h=1}^{r}, u=\sum k_{h} \ell_{h}} C_{K}\left(\prod_{h=1}^{r} t_{h}^{k_{h}} y^{n_{1} k_{h} j_{h}}\right)\left(1+y^{m n_{1}}\right)^{n_{2}-\sum_{h=1}^{r} k_{h}},
$$

for some $C_{K} \in \mathbb{Q}$, where $\delta_{q}^{u}$ is the Kronecker symbol. A similar formula holds for derivatives with respect to $y$ :

$$
\frac{\partial^{u} f_{\mathbf{t}, 2}}{\partial y^{u}}(x, 0)=\delta_{q}^{u} q ! x^{m a}+\sum_{K=\left(k_{h}\right)_{h=1}^{r}, u=\sum k_{h} \ell_{h}} C_{K}\left(\prod_{h=1}^{r} t_{h}^{k_{h}} x^{m k_{h} i_{h}}\right)\left(1+x^{m n_{1}}\right)^{n_{2}-\sum_{h=1}^{r} k_{h}},
$$


Let us compute the residue $\rho_{p}$. It is a linear combination with coefficients in $\mathbb{Q}$ of terms depending on couples $\left(V,\left(K_{w}\right)_{w}\right)$ where $V=\left(u_{w}\right) \in \mathcal{P}\left(\ell_{p}\right)$ and for each $w \in\{1, \ldots,|V|\}, K_{w}=\left(k_{h, w}\right)_{h=1}^{r}$ satisfies either

$$
\sum_{h=1}^{r} k_{h, w} \ell_{h}=u_{w}
$$

or the term involved is $y^{n_{1} b}$ or $x^{m a}$, i.e.,

$$
u_{w}=q, \quad k_{h, w}=0
$$

let $r_{V}$ be the number of terms of this type for $V$ then, the term is obtained as

$$
\begin{aligned}
& \int_{0}^{1} \prod_{w=1}^{|V|}\left(\prod_{h=1}^{r} t_{h}^{k_{h, w}} y^{n_{1} k_{h, w} j_{h}}\right) y^{r_{V} n_{1} b}\left(1+y^{m n_{1}}\right)^{\sum_{w}\left(n_{2}-\sum_{h=1}^{r} k_{h, w}\right)-n_{2}\left(\beta+|V|+r_{V}\right)} y^{n_{1}\left(1+j_{0}-j_{p}\right)} \frac{d y}{y}+ \\
& \int_{0}^{1} \prod_{w=1}^{|V|}\left(\prod_{h=1}^{r} t_{h}^{k_{h, w}} x^{m k_{h, w} i_{h}}\right) x^{r_{V} m a}\left(1+x^{m n_{1}}\right)^{\sum_{w}\left(n_{2}-\sum_{h=1}^{r} k_{h, w}\right)-n_{2}\left(\beta+|V|+r_{V}\right)} x^{m\left(1+i_{0}-i_{p}\right)} \frac{d x}{x} .
\end{aligned}
$$

This is a monomial in $t_{1}, \ldots, t_{r}$, namely,

$$
\prod_{h=1}^{r} t_{h}^{\sum_{w} k_{h, w}}
$$

whose coefficient is

$$
\begin{gathered}
\rho_{V,\left(K_{w}\right)}:=\int_{0}^{1} y^{n_{1}\left(\sum_{w, h} k_{h, w} j_{h}+1+j_{0}-j_{p}+r_{V} b\right)}\left(1+y^{m n_{1}}\right)^{-n_{2}\left(\beta+r_{V}\right)-\sum_{w, h} k_{h, w}} \frac{d y}{y}+ \\
\int_{0}^{1} x^{m\left(\sum_{w, h} k_{h, w} i_{h}+1+i_{0}-i_{p}+r_{V} a\right)}\left(1+x^{m n_{1}}\right)^{-n_{2}\left(\beta+r_{V}\right)-\sum_{w, h} k_{h, w}} \frac{d x}{x}= \\
G_{\left(1+y^{m n_{1}}\right)^{\alpha}}\left(n_{1}\left(\sum_{w, h} k_{h, w} j_{h}+1+j_{0}-j_{p}+r_{V} b\right)\right)+ \\
G_{\left(1+x^{m n_{1}}\right)^{\alpha}}\left(m\left(\sum_{w, h} k_{h, w} i_{h}+1+i_{0}-i_{p}+r_{V} a\right)\right)
\end{gathered}
$$

where

$$
\alpha:=-n_{2}\left(\beta+r_{V}\right)-\sum_{w, h} k_{h, w} .
$$

We need to compute the sum of the arguments

$$
\sigma:=n_{1}\left(\sum_{w, h} k_{h, w} j_{h}+1+j_{0}-j_{p}+r_{V} b\right)+m\left(\sum_{w, h} k_{h, w} i_{h}+1+i_{0}-i_{p}+r_{V} a\right) .
$$


From the equalities (A.4), (A.5) and the definition (A.2), we have

$$
u_{w}=m \sum_{h=1}^{r} k_{h, w} i_{h}+n_{1} \sum_{h=1}^{r} k_{h, w} j_{h}-m n_{1} \sum_{h=1}^{r} k_{h, w}
$$

if the first term is not involved and $u_{w}=q$ if it is; recall also that

$$
q=m a+n_{1} b-m n_{1} n_{2} .
$$

Then

$$
\begin{gathered}
i_{p} m+n_{1} j_{p}-m n_{1}=\ell_{p}=\sum_{w} u_{w}= \\
m\left(\sum_{w, h} k_{h, w} i_{h}+r_{V} a\right)+n_{1}\left(\sum_{w, h} k_{h, w} j_{h}+r_{V} b\right)-m n_{1}\left(\sum_{w, h} k_{h, w}+r_{V} n_{2}\right) .
\end{gathered}
$$

We obtain several properties from this equality. In particular

$$
\begin{gathered}
\sigma=m n_{1}\left(\sum_{w, h} k_{h, w}+r_{V} n_{2}\right)+\overbrace{n_{1}\left(1+j_{0}\right)+m\left(1+i_{0}\right)-m n_{1}}^{m+n_{1}+k=m n_{1} n_{2} \beta} \\
=m n_{1}\left(n_{2}\left(\beta+r_{V}\right)+\sum_{w, h} k_{h, w}\right)=-m n_{1} \alpha .
\end{gathered}
$$

By Lemma 1.5, we have that

$\rho_{V,\left(K_{w}\right)}=\frac{1}{m n_{1}} \boldsymbol{B}\left(\frac{\sum_{w, h} k_{h, w} j_{h}+1+j_{0}-j_{p}+r_{V} b}{m}, \frac{\sum_{w, h} k_{h, w} i_{h}+1+i_{0}-i_{p}+r_{V} a}{n_{1}}\right)$.

As another consequence from (A.8), we have that

$$
\frac{\sum_{w, h} k_{h, w} j_{h}-j_{p}+r_{V} b}{m}, \frac{\sum_{w, h} k_{h, w} i_{h}-i_{p}+r_{V} a}{n_{1}} \in \mathbb{Z} .
$$

Let us prove it. Since $\operatorname{gcd}\left(m, n_{1}\right)=1$, it is enough to show that the product of $n_{1}$ and the first denominator is congruent to $0 \bmod m$ :

$n_{1}\left(\sum_{w, h} k_{h, w} j_{h}+r_{V} b-j_{p}\right)=m\left(i_{p}-n_{1}-\sum_{w, h} k_{h, w} i_{h}-r_{V} a\right)+m n_{1}\left(\sum_{w, h} k_{h, w}+r_{V} n_{2}\right)$.

From the properties of the beta function, $\rho_{V,\left(K_{w}\right)}$ is a product of a non-zero rational number and $\boldsymbol{B}\left(\frac{1+i_{0}}{n_{1}}, \frac{1+j_{0}}{m}\right)$. As a consequence $\operatorname{Res}_{s=-\beta} I_{+}\left(f_{\mathbf{t}}, \beta_{1 i}, \beta_{2 i}\right)(s)$ is, up to the factor $\boldsymbol{B}\left(\frac{1+i_{0}}{n_{1}}, \frac{1+j_{0}}{m}\right)$ a polynomial $Q_{p}$ in the $t_{i}$ 's with coefficients in $\mathbb{Q}$; the coefficient of $t_{p}$ does not vanish. The only option to have the monomial $t_{p}$ is when $V=\left(\ell_{p}\right)$ and $K=\left(\ell_{p}\right), r_{V}=0$ and for these values

$$
\rho_{V,\left(K_{w}\right)}=\frac{1}{m n_{1}} \boldsymbol{B}\left(\frac{1+i_{0}}{n_{1}}, \frac{1+j_{0}}{m}\right) .
$$


Since $\ell_{1}$ is the minimum, $Q_{1}$ is a polynomial in $t_{1}$ of degree 1 . Then we can choose $t_{1}$ such that $\operatorname{Res}_{s=-\beta} I_{+}\left(f_{\mathbf{t}}, \beta_{11}, \beta_{21}\right)(s)=0$, since this residue is independent of $t_{p}$, for $p>1$. From now on $f_{\mathbf{t}}$ is a polynomial in $t_{2}, \ldots, t_{r}$, with $t_{1}$ fixed as above. In the same way, we choose $t_{2}$ such that

$$
\operatorname{Res}_{s=-\beta} I_{+}\left(f_{\mathbf{t}}, \beta_{12}, \beta_{22}\right)(s)=0,
$$

and recursively we can find $t_{3}, \ldots, t_{r}$ such that $\operatorname{Res}_{s=-\beta} I_{+}\left(f_{\mathbf{t}}, \beta_{1 p}, \beta_{2 p}\right)(s)=0$, for all $1 \leq p \leq r$ and all the $t$ 's are in $\mathbb{Q}$. Using Proposition 1.7 it is easy to proof that $f_{\mathbf{t}}$ is of type $\left(n_{1} n_{2}, m n_{2}, m n_{2}+q\right)^{+}$.

Proof of Claim 2.10. Let $\left(\beta_{1}^{\prime}, \beta_{2}^{\prime}, \beta_{3}\right)$ be as in the statement. If $n_{2}\left(\beta_{1}^{\prime} m+\beta_{2}^{\prime} n_{1}\right)+$ $\beta_{3}\left(n_{1} n_{2} m+q\right)>n_{2}\left(m+n_{1}\right)+k$, it is not hard to check that the statement holds trivially for any $f$ of type $\left(n_{1} n_{2}, m n_{2}, m n_{2}+q\right)^{-}$.

We are going to characterize the triples not satisfying the above inequality and to find an $f_{\beta}$ satisfying the conditions of the statement. Let

$$
M_{\beta}=\left\{\left(\tilde{\beta}_{1}, \tilde{\beta}_{2}, \beta_{3}, \nu\right) \in \mathbb{Z}_{\geq 0}^{3} \times \mathbb{Z}_{\geq 1} \mid k=n_{2}\left(m \tilde{\beta}_{1}+n_{1} \tilde{\beta}_{2}\right)+\left(m n_{1} n_{2}+q\right) \beta_{3}+\nu\right\} .
$$

It is not hard to prove the following properties:

- if $\left(\tilde{\beta}_{1}, \tilde{\beta}_{2}, \beta_{3}, \nu\right) \in M_{\beta}$, then $\beta_{3}<n_{2}$;

- if moreover $\left(\tilde{\beta}_{1}^{\prime}, \tilde{\beta}_{2}^{\prime}, \beta_{3}^{\prime}, \nu\right) \in M_{\beta}$ then $\beta_{3}=\beta_{3}^{\prime}$.

We denote by $N_{\beta}$ the set of $\nu$ which are the fourth coordinate of some element of $M_{\beta}$ and we order $N_{\beta}$. For $\nu \in N_{\beta}$, choose $\tilde{\beta}_{1}, \tilde{\beta}_{2}, \beta_{3}$ such that $\left(\tilde{\beta}_{1}, \tilde{\beta}_{2}, \beta_{3}, \nu\right) \in M_{\beta}$; if we denote $\beta_{i}=\tilde{\beta}_{i}+1, i=1,2$, we have:

$$
n_{2}\left(m+n_{1}\right)+k=n_{2}\left(m \beta_{1}+n_{1} \beta_{2}\right)+\left(m n_{1} n_{2}+q\right) \beta_{3}+\nu .
$$

Note that $\beta_{3}$ is determined by $\nu$; it may not be the case for $\beta_{1}, \beta_{2}$. Let $\ell_{\nu}$ such that $0 \leq \ell_{\nu}<n_{2}$, and $a_{\nu}, b_{\nu} \in \mathbb{Z}_{\geq 0}$ such that

$$
\left(m n_{1} n_{2}+q\right) \ell_{\nu}+\left(m a_{\nu}+n_{1} b_{\nu}\right) n_{2}=\left(m n_{1} n_{2}+q\right) n_{2}+\nu
$$

Let

$$
f_{\beta}(x, y)=\left(x^{n_{1}}-y^{m}\right)^{n_{2}}+x^{a} y^{b}+\sum_{\nu \in N_{\beta}} t_{\nu}\left(x^{n_{1}}-y^{m}\right)^{\ell_{\nu}} x^{a_{\nu}} y^{b_{\nu}}
$$

We choose $f_{\beta}$ of type $\left(n_{1} n_{2}, m n_{2}, m n_{2}+q\right)^{-}$. Let us recall the change of variables that allows to compute the poles of the proper integrals. Note that in this case, 
one can choose $g_{Y}=x^{n_{1}}-y^{m}$. We have:

$$
\begin{gathered}
\tilde{f}_{\beta}(x, y)=\left(x^{m n_{1}}-y^{m n_{1}}\right)^{n_{2}}+x^{m a} y^{n_{1} b}+\sum_{\nu \in N_{\beta}} t_{\nu}\left(x^{m n_{1}}-y^{m n_{1}}\right)^{\ell_{\nu}} x^{m a_{\nu}} y^{n_{1} b_{\nu}} \\
\tilde{\tilde{f}}_{\beta}(x, y)=\left(1-y^{m n_{1}}\right)^{n_{2}}+x^{q} y^{n_{1} b}+\sum_{\nu \in N_{\beta}} t_{\nu}\left(1-y^{m n_{1}}\right)^{\ell_{\nu}} x^{\frac{\nu+q\left(n_{2}-\ell_{\nu}\right)}{n_{2}}} y^{n_{1} b_{\nu}} \\
\hat{f}_{\beta}(x, y)=y^{n_{2}} h_{1}(y)+x^{q} h_{2}(y)+\sum_{\nu \in N_{\beta}} t_{\nu} y^{\ell_{\nu}} h_{3, \nu}(y) x^{\frac{\nu+q\left(n_{2}-\ell_{\nu}\right)}{n_{2}}} \\
\hat{\hat{f}}_{\beta}(x, y)=y^{n_{2} q} h_{1}\left(y^{q}\right)+x^{n_{2} q} h_{2}\left(y^{q}\right)+\sum_{\nu \in N_{\beta}} t_{\nu} y^{\ell_{\nu} q} h_{3, \nu}\left(y^{q}\right) x^{\nu+q\left(n_{2}-\ell_{\nu}\right)} \\
f_{1 \beta}(x, y)=y^{n_{2} q} h_{1}\left(x^{q} y^{q}\right)+h_{2}\left(x^{q} y^{q}\right)+\sum_{\nu \in N_{\beta}} t_{\nu} y^{\ell_{\nu} q} h_{3, \nu}\left(x^{q} y^{q}\right) x^{\nu} \\
f_{2 \beta}(x, y)=h_{1}\left(y^{q}\right)+x^{n_{2} q} h_{2}\left(y^{q}\right)+\sum_{\nu \in N_{\beta}} t_{\nu} y^{\nu} h_{3, \nu}\left(y^{q}\right) x^{\nu+q\left(n_{2}-\ell_{\nu}\right)}
\end{gathered}
$$

where $h_{1}(0)=\left(m n_{1}\right)^{n_{2}}, h_{2}(0)=1$ and $h_{3, \nu}(0)=\left(m n_{1}\right)^{\ell_{\nu}}$, deg $h_{3}(y)=\left(m n_{1}-\right.$ 1) $\ell_{\nu}+n_{1} b_{\nu}$. For further use, $c_{i j}$ is the coefficient of $y^{j}$ in $h_{i}, i=1,2$ and $c_{3, \nu, j}$ for $h_{3, \nu}$.

Let

$$
\tilde{g}(x, y)=x^{m n_{1}}-y^{m n_{1}}, \tilde{\tilde{g}}(x, y)=1-y^{m n_{1}}
$$

and define $h_{4}(y)$ by the property

$$
y^{\beta_{3}} h_{4}(y)=\left(1-(1-y)^{m n_{1}}\right)^{\beta_{3}}(1-y)^{n_{1} \beta_{2}-1},
$$

where $h_{4}(0)=\left(m n_{1}\right)^{\beta_{3}}$, and write

$$
h_{4}(y)=\sum_{i=1}^{\left(m n_{1}-1\right) \beta_{3}+n_{1} \beta_{2}} b_{i} y^{i-1} .
$$

We want to compute $\operatorname{Res}_{s=-\beta} I_{-}\left(f_{\beta}, \beta_{1}, \beta_{2}, \beta_{3}\right)(s)$. For $1 \leq i \leq\left(m n_{1}-1\right) \beta_{3}+n_{1} \beta_{2}$, set $\nu_{i}$ such that

$$
\beta=\frac{n_{2}\left(m \beta_{1}+n_{1} \beta_{2}+m n_{1} \beta_{3}\right)+q\left(\beta_{3}+i\right)+\nu_{i}}{n_{2}\left(m n_{1} n_{2}+q\right)} ;
$$

we dismiss the cases where $\nu_{i}<0$; note that $\nu=i q+\nu_{i}$. The formula for the residue, see (1.9), is:

$$
\begin{gathered}
\operatorname{Res}_{s=-\beta} I_{-}\left(f_{\beta}, \beta_{1}, \beta_{2}, \beta_{3}\right)(s)= \\
\frac{1}{n_{2} q} \sum_{i} \frac{1}{\nu_{i} !} b_{i}\left(G_{h_{\nu_{i},-\beta, x}^{1}}\left(q\left(\beta_{3}+i\right)\right)+G_{h_{\nu_{i},-\beta, y}^{2}}\left(n_{2}\left(m \beta_{1}+n_{1} \beta_{2}+m n_{1} \beta_{3}-m n_{1} n_{2} \beta\right)\right)\right.
\end{gathered}
$$


where

$$
h_{\nu_{i},-\beta, x}^{1}(y)=\frac{\partial^{\nu_{i}} f_{1 \beta}^{-\beta}}{\partial x^{\nu_{i}}}(0, y), \quad h_{\nu_{i},-\beta, y}^{2}(x)=\frac{\partial^{\nu_{i}} f_{2 \beta}^{-\beta}}{\partial y^{\nu_{i}}}(x, 0) .
$$

We proceed as in the proof of Claim 2.8:

$$
\begin{aligned}
& \frac{\partial^{\nu_{i}} f_{1 \beta}^{-\beta}}{\partial x^{\nu_{i}}}(0, y)=\sum_{V=\left(\nu_{w}\right) \in \mathcal{P}\left(\nu_{i}\right)} D_{V}\left(\prod_{w=1}^{|V|} \frac{\partial^{\nu_{w}} f_{1 \beta}}{\partial x^{\nu_{w}}}(0, y)\right)\left(\left(m n_{1}\right)^{n_{2}} y^{n_{2} q}+1\right)^{-\beta-|V|} \\
& \frac{\partial^{\nu_{i}} f_{2 \beta}^{-\beta}}{\partial y^{\nu_{i}}}(x, 0)=\sum_{V=\left(\nu_{w}\right) \in \mathcal{P}\left(\nu_{i}\right)} D_{V}\left(\prod_{w=1}^{|V|} \frac{\partial^{\nu_{w}} f_{2 \beta}}{\partial y^{\nu_{w}}}(x, 0)\right)\left(\left(m n_{1}\right)^{n_{2}}+x^{n_{2} q}\right)^{-\beta-|V|}
\end{aligned}
$$

with $D_{V} \in \mathbb{Q}$. The derivatives without powers are computed as follows. For $u \in \mathbb{Z}_{\geq 0}$, let $q_{u}:=\left\lfloor\frac{u}{q}\right\rfloor$ and set

$$
\begin{gathered}
\mathcal{C}(u):=\left\{h \in\left\{0,1, \ldots, q_{u}\right\} \mid u-q h=\nu_{h} \in N_{\beta}\right\} \\
\frac{1}{u !} \frac{\partial^{u} f_{1 \beta}}{\partial x^{u}}(0, y)=\sum_{h \in \mathcal{C}(u)} c_{3, \nu_{h}, h} t_{\nu_{h}} y^{\left(\ell_{\nu_{h}}+h\right) q}+\chi_{\mathbb{Z}}\left(K_{u}\right)\left(c_{K_{u} 1} y^{n_{2} q}+c_{K_{u} 2}\right) y^{u} \\
\frac{1}{u !} \frac{\partial^{u} f_{2 \beta}}{\partial y^{u}}(x, 0)=\sum_{h \in \mathcal{C}(u)} c_{3, \nu_{h}, h} t_{\nu_{h}} x^{\nu_{h}+q\left(n_{2}-\ell_{\nu_{h}}\right)}+\chi_{\mathbb{Z}}\left(K_{u}\right)\left(c_{K_{u} 1}+c_{K_{u} 2} x^{n_{2} q}\right)
\end{gathered}
$$

where $K_{u}=\frac{u}{q}$ and $\chi_{\mathbb{Z}}$ is the characteristic function of $\mathbb{Z}$.

The terms of the derivatives involved in the computation of the residues are parametrized by $V=\left(\nu_{w}\right)_{w=1}^{|V|} \in \mathcal{P}\left(\nu_{i}\right)$; given $V$ we decompose its set of indices in three parts:

- $w \in W_{1}$, which determines $h_{w} \in \mathcal{C}\left(\nu_{w}\right)$, corresponding to a term with coefficient $c_{3, \nu_{h_{w}}, h_{w}}$;

- $w \in W_{2}$, where $\nu_{w} \equiv 0 \bmod q$, corresponding to a term with coefficient $c_{K_{\nu_{w}}}$;

- $w \in W_{3}$, where $\nu_{w} \equiv 0 \bmod q$, corresponding to a term with coefficient $c_{K_{\nu_{w}}}$,

where $W=W_{1} \coprod W_{2} \coprod W_{3}$. For such a 4 -uple $\left(V, W_{1}, W_{2}, W_{3}\right)$ the integrands are

$$
\begin{aligned}
& \left(\prod_{w \in W_{1}} t_{\nu_{h_{w}}} y^{\left(\ell_{\nu_{w}}+h_{w}\right) q}\right)\left(\prod_{w \in W_{2}} y^{\nu_{w}+n_{2} q}\right)\left(\prod_{w \in W_{3}} y^{\nu_{w}}\right)\left(\left(m n_{1}\right)^{n_{2}} y^{n_{2} q}+1\right)^{-\beta-|V|} y^{q\left(\beta_{3}+i\right)}= \\
& \left(\prod_{w \in W_{1}} t_{\nu_{h_{w}}}\right) y^{q\left(\sum_{w \in W_{1}}\left(\ell_{\nu_{h_{w}}}+h_{w}\right)+\sum_{w \in W_{2} \cup W_{3}} K_{\nu_{w}}+n_{2}\left|W_{2}\right|+\beta_{3}+i\right)}\left(\left(m n_{1}\right)^{n_{2}} y^{n_{2} q}+1\right)^{-\beta-|V|}
\end{aligned}
$$


and

$$
\begin{aligned}
& \left(\prod_{w \in W_{1}} t_{\nu_{h_{w}}} x^{\nu_{h_{w}}+q\left(n_{2}-\ell_{\nu_{h_{w}}}\right)}\right)\left(\prod_{w \in W_{3}} x^{n_{2} q}\right)\left(\left(m n_{1}\right)^{\left.n_{2}+x^{n_{2} q}\right)^{-\beta-|V|}} x^{n_{2}\left(m \beta_{1}+n_{1} \beta_{2}+m n_{1} \beta_{3}-m n_{1} n_{2} \beta\right)}=\right. \\
& \left(\prod_{w \in W_{1}} t_{\nu_{h_{w}}}\right) x^{\sum_{w \in W_{1}}\left(\nu_{h_{w}}+q\left(n_{2}-\ell_{\nu_{w}}\right)\right)+n_{2}\left(q\left|W_{3}\right|+m \beta_{1}+n_{1} \beta_{2}+m n_{1} \beta_{3}-m n_{1} n_{2} \beta\right)}\left(\left(m n_{1}\right)^{\left.n_{2}+x^{n_{2} q}\right)^{-\beta-|V|}}\right.
\end{aligned}
$$

If $\alpha=-\beta-|V|$, we need to compute

$$
\begin{aligned}
& \left.G_{\left(\left(m n_{1}\right)^{n_{2}} y^{n_{2} q}+1\right)^{\alpha}}\left(q\left(\sum_{w \in W_{1}}\left(\ell_{\nu_{h_{w}}}+h_{w}\right)+\sum_{w \in W_{2} \cup W_{3}} K_{\nu_{w}}+n_{2}\left|W_{2}\right|+\beta_{3}+i\right)\right)\right)+
\end{aligned}
$$

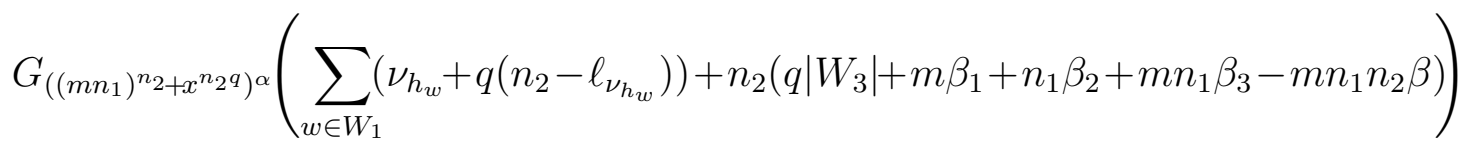

The sum of the two entries equals $-n q_{2} \alpha$, and by Lemma 1.5, we have that this contribution equals

$$
\frac{\left(m n_{1}\right)^{\sum_{w \in W_{1}}\left(\ell_{h_{w}}+h_{w}\right)+\sum_{w \in W_{2} \cup W_{3}} K_{\nu_{w}}+n_{2}\left|W_{2}\right|+\beta_{3}+i}}{n_{2} q} \boldsymbol{B}(u, v)
$$

where

$$
\begin{gathered}
u:=\frac{1}{n_{2}}\left(\sum_{w \in W_{1}}\left(\ell_{\nu_{h_{w}}}+h_{w}\right)+\sum_{w \in W_{2} \cup W_{3}} K_{\nu_{w}}+n_{2}\left|W_{2}\right|+\beta_{3}+i\right) \\
v:=\frac{1}{n_{2} q} \sum_{w \in W_{1}}\left(\nu_{h_{w}}+q\left(n_{2}-\ell_{\nu_{h_{w}}}\right)\right)+\frac{1}{q}\left(q\left|W_{3}\right|+m \beta_{1}+n_{1} \beta_{2}+m n_{1} \beta_{3}-m n_{1} n_{2} \beta\right)
\end{gathered}
$$

Note that $u+v=\beta+|V|$, i.e., it is congruent with $\beta \bmod \mathbb{Z}$.

On the other side, since $q \ell_{\nu} \equiv \nu \bmod n_{2}$ the following congruences $\bmod n_{2}$ hold:

$$
\begin{gathered}
q\left(n_{2} u-\left(\ell_{\nu}+\beta_{3}\right)\right) \equiv \sum_{w \in W_{1}} q\left(\ell_{\nu_{h_{w}}}+h_{w}\right)+\sum_{w \in W_{2} \cup W_{3}} \nu_{w}+q i-q \ell_{\nu} \equiv \\
\sum_{w \in W_{1}}\left(\nu_{h_{w}}+q h_{w}\right)+\sum_{w \in W_{2} \cup W_{3}} \nu_{w}+q i-\nu \equiv \sum_{w=1}^{|V|} \nu_{w}+q i-\nu=\nu_{i}+q i-\nu=0 ;
\end{gathered}
$$

since $\operatorname{gcd}\left(q, n_{2}\right)=1$, we deduce that

$$
u-\frac{\ell_{\nu}+\beta_{3}}{n_{2}} \in \mathbb{Z}
$$

In particular, the corresponding contribution is, up to a factor in $\mathbb{Q}$, the value $\boldsymbol{B}\left(\frac{\ell_{\nu}+\beta_{3}}{n_{2}}, \beta-\frac{\ell_{\nu}+\beta_{3}}{n_{2}}\right)$. Hence, the total result is the product of this value and a polynomial in t with coefficients in $\mathbb{Q}$ and the coefficients of degree 1 do not vanish. 
Let us denote $N_{\beta}=\left\{\left(\nu^{(1)}, \ldots, \nu^{(r)}\right\}\right.$ where $\nu^{(1)}<\cdots<\nu^{(r)}$. For $\nu^{(1)}$, let us consider the corresponding $\beta_{1}^{(1)}, \beta_{2}^{(1)}, \beta_{3}^{(1)}$. Then,

$$
\operatorname{Res}_{s=-\beta} I_{-}\left(f_{\beta}, \beta_{1}^{(1)}, \beta_{2}^{(1)}, \beta_{3}^{(1)}\right)(s)=\boldsymbol{B}\left(\frac{\ell_{\nu^{(1)}}+\beta_{3}}{n_{2}}, \beta-\frac{\ell_{\nu^{(1)}}+\beta_{3}}{n_{2}}\right) q^{(1)}\left(t_{1}\right)
$$

where $q^{(1)}$ is of degree 1 . Hence, we can choose $t_{1} \in \mathbb{R}$ such that the above residue vanishes. Recursively, we can choose $t_{1}, \ldots, t_{r} \in \mathbb{R}$ such that $f_{\beta}$ is of type $\left(n_{1} n_{2}, m n_{2}, m n_{2}+q\right)^{-}$and

$$
\operatorname{Res}_{s=-\beta} I_{-}\left(f_{\beta}, \beta_{1}^{(j)}, \beta_{2}^{(j)}, \beta_{3}^{(j)}\right)(s)=0, \quad 1 \leq j \leq r .
$$

This result does not depend on the particular choice of $\left(\beta_{1}^{(j)}, \beta_{2}^{(j)}\right)$.

\section{REFERENCES}

[1] E. Artal Bartolo, Pi. Cassou-Noguès, I. Luengo and A. Melle-Hernández, Yano's conjecture for 2-Puiseux pairs irreducible plane curve singularities, to appear in Publications of the RIMS, available at arXiv:1602.07248 [math.AG], Preprint 2016.

[2] I.N. Bernšteĭn, Analytic continuation of generalized functions with respect to a parameter, Funkcional. Anal. i Priložen. 6 (1972), no. 4, 26-40.

[3] J.-E. Björk, The Bernstein class of modules on algebraic manifolds, Paul Dubreil and MariePaule Malliavin Algebra Seminar, 33rd Year (Paris, 1980), Lecture Notes in Math., vol. 867, Springer, Berlin-New York, 1981, pp. 148-156.

[4] Pi. Cassou-Noguès, Racines de polyômes de Bernstein, Ann. Inst. Fourier (Grenoble) 36 (1986), no. 4, 1-30.

[5] Pi. Cassou-Noguès, Séries de Dirichlet et intégrales associées à un polynôme à deux indéterminées, J. Number Theory 23 (1986), no. 1, 1-54.

[6] Pi. Cassou-Noguès, Étude du comportement du polynôme de Bernstein lors d'une déformation à $\mu$-constant de $X^{a}+Y^{b}$ avec $(a, b)=1$, Compositio Math. 63 (1987), no. 3, 291-313.

[7] Pi. Cassou-Noguès, Polynôme de Bernstein générique, Abh. Math. Sem. Univ. Hamburg 58 (1988), 103-123.

[8] S.C. Coutinho, A primer of algebraic D-modules, London Mathematical Society Student Texts, vol. 33, Cambridge University Press, Cambridge, 1995.

[9] W. Decker, G.-M. Greuel, G. Pfister and H. Schönemann, Singular 4-0-2 - A computer algebra system for polynomial computations, http://www.singular.uni-kl.de, 2015.

[10] A.G. Guimaraes and A. Hefez, Bernstein-Sato polynomials and spectral numbers, Ann. Inst. Fourier (Grenoble) $5 \mathbf{7}$ (2007), no. 6, 2031-2040.

[11] C. Hertling and C. Stahlke, Bernstein polynomial and Tjurina number, Geom. Dedicata 75 (1999), p. 137-176.

[12] M. Kashiwara, B-functions and holonomic systems. Rationality of roots of B-functions, Invent. Math. 38 (1976/77), no. 1, 33-53.

[13] H.B. Laufer, The multiplicity of isolated two-dimensional hypersurface singularities, Trans. Amer. Math. Soc. 302, (1987), no. 2, p. 489-496. 
[14] V. Levandovskyy, and J. Martín-Morales, Algorithms for checking rational roots of bfunctions and their applications, J. Algebra 352 (2012), p. 408-429.

[15] B. Lichtin, Poles of $|f(z, w)|^{2 s}$ and roots of the b-function, Ark. Mat. 27 (1989), no. 2, 283-304.

[16] F. Loeser, Fonctions d'Igusa p-adiques et polynômes de Bernstein, Amer. J. Math. 110 (1988), 1-21.

[17] I. Luengo and G. Pfister, Normal forms and moduli spaces of curve singularities with semigroup $\langle 2 p, 2 q, 2 p q+d\rangle$, Compositio Math. 76 (1990), no. 1-2, p. 247-264.

[18] B. Malgrange, Le polynôme de Bernstein d'une singularité isolée, Fourier integral operators and partial differential equations (Colloq. Internat., Univ. Nice, Nice, 1974), Springer, Berlin, 1975, pp. 98-119. Lecture Notes in Math., Vol. 459.

[19] A. Némethi, On the spectrum of curve singularities, Proceedings of the Singularity Conference, Oberwolfach, July 1996, Progress in Mathematics, vol 162, 1998, p. 93-102.

[20] W.D. Neumann, A calculus for plumbing applied to the topology of complex surface singularities and degenerating complex curves, Trans. Amer. Math. Soc. 268 (1981), no. 2, p. 299-344.

[21] M. Saito, On the structure of Brieskorn lattice, Ann. Inst. Fourier (Grenoble) 39 (1989), no. $1,27-72$.

[22] M. Saito, On b-function, spectrum and rational singularity, Math. Ann. 295 (1993), p. $51-74$.

[23] M. Saito, Exponents of an irreducible plane curve singularity, Preprint available at arXiv:009133 [math.AG], 2000.

[24] R. Schrauwen, J.H.M. Steenbrink and J. Stevens, Spectral pairs and the topology of curve singularities, Complex geometry and Lie theory (Sundance, UT, 1989),, Proc. Sympos. Pure Math., 53, Amer. Math. Soc., Providence, RI, 1991, p. 305-328.

[25] J.H.M. Steenbrink, Mixed Hodge structure on the vanishing cohomology, Real and complex singularities (Proc. Ninth Nordic Summer School/NAV, Sympos. Math., Oslo, 1976) Sijthoff and Noordhoff, Alphen aan den Rijn, 1977, p. 525-563.

[26] W.A. Stein and others, Sage Mathematics Software (Version 7.3), The Sage Development Team, 2016, http://www.sagemath.org.

[27] A.N. Varčenko, Gauss-Manin connection of isolated singular point and Bernstein polynomial, Bull. Sci. Math. (2) 104 (1980), no. 2, 205-223.

[28] A. N. Varčenko, The complex singularity index does not change along the stratum $\mu=\mathrm{const}$, Funktsional. Anal. i Prilozhen. 16 (1982), no. 1, p. 1-12.

[29] T. Yano, Exponents of singularities of plane irreducible curves, Sci. Rep. Saitama Univ. Ser. A 10 (1982), no. 2, 21-28. 
Departamento de Matemáticas-IUma, Universidad de Zaragoza, c/Pedro CerBUNA 12, 50009 ZARAGOZA, SPAIN

E-mail address: artal@unizar.es

Institut de Mathématiques de Bordeaux, Université de Bordeaux , 350, Cours De la Libération, 33405, Talence Cedex 05, FRANCE

E-mail address: Pierrette.Cassou-nogues@math.u-bordeaux.fr

Departamento de Álgebra, Universidad Complutense, Plaza de las Ciencias S/n, Ciudad Universitaria, 28040 Madrid, SPAIN

E-mail address: iluengo@ucm.es

Departamento de Álgebra, Universidad Complutense, Plaza de las Ciencias S/n, CiUdad Universitaria, 28040 Madrid, SPAIN

E-mail address: amelle@ucm.es 